\title{
A Survey of Rigidity Theory for Anosov Actions Steven Hurder
}

\author{
Abstract \\ We survey the recent results on the topological and smooth classifica- \\ tion of Anosov group actions, and present some of the many interesting \\ questions which remain open in this field.
}

\section{Introduction - the main conjecture}

"Rigidity" for a given dynamical system means that every nearby dynamical system (usually in the $C^{1}$-topology on maps) is topologically equivalent to the initial system. One cannot expect the conjugacy to be differentiable, because there are linear invariants associated to periodic points which are not invariant under $C^{1}$-perturbation. Smale's paper [59] laid the foundations for this theory some 25 years ago, and the subject has seen remarkable developments since then, especially for hyperbolic dynamical systems. For the the non-hyperbolic case, the subject is still in development, as pointed out in the opening talk by J. Palis at this conference.

Rigidity theory for group actions has developed in response to problems from geometry and Lie Group theory, more than from dynamics. The problem is roughly the same: prove that a nearby system, usually in the $C^{1}$-topology on the generators of the action, is topologically equivalent to the initial action. However, in the case when the group has additional algebraic structure, it is possible to demand that more be shown; for example, that the conjugacy be smooth or real analytic. In certain cases, one can prove a classification result, that all actions of a certain type originate from an algebraic construction. This is the content of the celebrated Mostow rigidity theorem for actions of higher rank lattices on appropriate symmetric spaces, as well as the Franks-NewhouseManning classification theory of Anosov abelian actions on nilmanifolds.

\footnotetext{
AMS classification: Primary 58G25, 57R30, 58F18, 35P15

Supported in part by NSF Grant DMS 91-03297

This paper is in final form and no version of it will be submitted for publication elsewhere.
} 
There is a third class of group actions for which smooth and analytic conjugacy to an algebraic action can be proven: when the group is a "higher rank lattice", and the action is "irreducible", Anosov and volume-preserving on a tori or nil-manifold. In this survey we will introduce the reader to the rapidly developing research on the rigidity phenomenon possessed by actions of higher rank lattices on tori and nilmanifolds. There are many techniques in current use, and more being developed with time, as well as many open questions to ponder. Questions about understanding the actions of higher rank lattices on tori were raised at a conference on the topic of "Ergodic Theory, Lie Groups and Geometry" at the Mathematical Sciences Research Institute, Berkeley in May 1984. R. Zimmer discussed his (then) recent work on the rigidity of isometric actions of higher rank lattices on compact manifolds [72], and posed the problem of obtaining similar results for non-isometric actions. Perturbations of the standard action of $S L(n, \mathbf{Z})$ on $\mathbf{T}^{n}$ give the canonical example of this type, and it was asked whether they must be conjugate to the standard action. D. Dried contributed a key remark during these discussions, that the existence of hyperbolic matrices in $S L(n, \mathbf{Z})$ ought to be of use. The problem set [24] and Zimmer's papers $[73,75]$ discuss some of the open questions at that time.

Many of the same conferencers met for a workshop on "Geometric Rigidity" at the University of Colorado in Boulder, May 1989, at which many interesting and exciting results were presented. Among the talks was a report by J. Lewis on the main theorem of his thesis, that for $n \geq 7$, all infinitesimal variations of the standard action are trivial [37]. This translates into a statement about the vanishing of the first cohomology group for $S L(n, \mathbf{Z})$ with coefficients in the module of smooth vector fields on $\mathbf{T}^{n}$. Lewis proved that work of Zimmer on the vanishing of the first cohomology with coefficients in the measurable vector fields [76], which had evolved from the techniques of his isometric rigidity theorems, could be improved to the smooth case. It was remarked at the time, by the author and A. Katok, that Lewis' methods were strongly reminiscent of the regularity theorem (Lemma 2.3, [43]; Theorem 2.6, [31]) which had been used in hyperbolic dynamics to prove the Livsic Theorem [38, 39] that certain measurable cocycles (i.e., a special class of functions on the manifold) were necessarily smooth.

Two new aspects concerning the rigidity of lattice actions on tori were obtained by the author in August, 1989, and published in the announcement [25] and paper [28]: given a $C^{1}$-continuous path of such actions, if the initial action has a "hyperbolic element" and the periodic points for the action are dense, then each action in the path is topologically conjugate to the initial action, for small time at least. This is called topological deformation rigidity for the action, and holds very generally (see Theorem 2.3 below.) Secondly, a topological conjugacy between actions with a large commuting "sufficiently hyperbolic" subgroup is necessarily as smooth as the actions (see Theorem 3.14.) Thus, given a topological solution of the rigidity problem, very often the group structure and the dynamics force the conjugacy to be smooth or even real analytic. 
A. Katok and J. Lewis announced at a conference on "Geometric Rigidity" at Northwestern University, May 1990, the result that a $C^{1}$-perturbation of the standard action of $S L(n, \mathbf{Z})$ on $\mathbf{T}^{n}$ is topologically conjugate to the standard action for $n>4$. This was later improved to hold for $n \geq 4$, with the proofs appearing in [34]. Combined with the regularity theory that topological conjugacy implies smooth conjugacy, this settled Zimmer's initial question for $n \geq 4$.

Subsequent developments have engulfed many related questions, and further techniques were employed in the study of the problem, especially the cocycle super-rigidity theory of R. Zimmer [68, 69]. This has lead to global rigidity results, where the smooth algebraic structure can be deduced without assuming that the given action is $C^{1}$-perturbation of an algebraic action. Cocycle superrigidity was applied by A. Katok, J. Lewis and R. Zimmer in [35] to reprove the perturbation rigidity for actions of finite-index subgroups of $S L(n, \mathbf{Z})$ on $\mathbf{T}^{n}$, including the case $n=3$. The author used cocycle super-rigidity and the methods of [28] to prove global rigidity for a large class of lattice actions in [29], as described in Theorem 4.2 below. M. Pollicott has recently [54] given a more direct proof of the topological rigidity of the standard action of $S L(n, \mathbf{Z})$ acting on $\mathbf{T}^{n}$.

The success of the rigidity program for lattice actions has spurred the investigation into other rigidity phenomenon associated to Anosov actions. For example, the recent remarkable work of Katok and Spatzier [36] have yielded a much deeper insight into the smooth classification of Anosov actions of abelian groups, extending the Franks-Newhouse-Manning theory.

The model examples for the classification results are all derived from the following simple constructions. The natural action of the determinant-one, integer $n \times n$ matrices $S L(n, \mathbf{Z})$ on $\mathbf{R}^{n}$ preserves the integer lattice $\mathbf{Z}^{n}$, hence for each subgroup $\Gamma \subset S L(n, \mathbf{Z})$ there is an induced "standard action" on the quotient $n$-torus,

$$
\varphi: \Gamma \times \mathbf{T}^{n} \rightarrow \mathbf{T}^{n} .
$$

If $\Gamma$ contains a hyperbolic matrix $A \in \Gamma$, then we say that $\varphi$ is an Anosov action. This example admits a natural generalization to lattice actions on nilmanifolds (cf. [9]), and there are non-standard affine actions of lattices (cf. section 6, or [27]) obtained from this basic model by twisting the action with a translational cocycle. Katok and Lewis have constructed non-affine actions of higher rank lattices (cf. Example 8 below), suggesting the possibility that there exist nonaffine Anosov actions.

Let us introduce the general formulation of our rigidity program. Let $X$ denote a compact $C^{r}$-manifold without boundary of dimension $n$ with $1 \leq r \leq$ $\infty$. We set $r=\omega$ for the case of a real analytic manifold and real analytic actions. A $C^{r}$-action $\varphi: \Gamma \times X \rightarrow X$ of a group $\Gamma$ on a $X$ is said to be Anosov if there exists at least one element, $\gamma_{h} \in \Gamma$, such that $\varphi\left(\gamma_{h}\right)$ is an Anosov diffeomorphism [2] of $X$. We say that $\gamma$ is $\varphi$-hyperbolic. 
A finitely-generated group $\Gamma$ is a said to be a higher rank lattice if $\Gamma$ is a discrete subgroup of a connected semi-simple algebraic $\mathbf{R}$-group $G$, with the $\mathbf{R}$-split rank of each factor of $G$ at least $2, G$ has finite center and $G_{\mathbf{R}}^{0}$ has no compact factors, so that $G / \Gamma$ has finite volume.

The strongest form of rigidity is that there is a set of algebraic models for the class of group actions under study, and a given action is topologically conjugate to one of these, which is determined by a finite set of algebraic or topological invariants of the action. In our context, the models are derived from the fundamental group of the manifold, and the action is determined by its homotopy type:

CONJECTURE 1.1 (Anosov Rigidity) Let $\Gamma$ be a lattice of higher rank, and $\varphi: \Gamma \times X \rightarrow X$ a $C^{r}$-Anosov action on a compact smooth manifold $X$ of dimension $n$, for $1 \leq r \leq \omega$. Then:

1. There is a finite covering of $X$ by a nilmanifold $\tilde{X}$;

2. There is a subgroup $\tilde{\Gamma} \subset \Gamma$ of finite index for which the action $\varphi \mid \tilde{\Gamma}$ lifts to an action $\tilde{\varphi}$ on $\tilde{X}$ with a fixed point;

3. The $C^{r}$-conjugacy class of $\tilde{\varphi} \mid \tilde{\Gamma}$ is determined by the homotopy type of the action. That is, $\tilde{\varphi}$ is topologically conjugate to the standard algebraic action of $\tilde{\Gamma}$ induced on the nilmanifold $\hat{\pi}_{1}(\tilde{X}) / \pi_{1}(\tilde{X})$, where $\hat{\pi}_{1}(\tilde{X})$ denotes the Mal'cev completion of the fundamental group of $\tilde{X}$.

\section{Topological rigidity of Anosov actions}

In this section, we discuss the known results on the topological rigidity of lattice actions. There are three properties of the standard action of $S L(n, \mathbf{Z})$ on $\mathbf{T}^{n}$ which we abstract to a general $C^{1}$-action : the presence of a hyperbolic element, as introduced in the last section; density of the periodic orbits; and some form of cohomology vanishing theorem.

A point $x \in X$ is periodic for $\varphi$ if the set

$$
\Gamma(x) \stackrel{\text { def }}{=}\{\varphi(\gamma)(x) \mid \gamma \in \Gamma\}
$$

is finite. Let $\Lambda=\Lambda(\varphi) \subset X$ denote the set of periodic points for $\varphi$. For each $x \in \Lambda$, let $\Gamma_{x} \subset \Gamma$ denote the isotropy subgroup of $x$. Note that the index $\left[\Gamma, \Gamma_{x}\right] \leq o(x)$ ! where $o(x)=|\Gamma(x)|$ is the order of the orbit of $x$.

The cohomology condition can be imposed universally on the group $\Gamma$, or can be formulated more particularly in terms of the given action. The basic idea is that we need to have the conclusion of the stability theorem of D. Stowe for lattice actions with a periodic point $[60,61]$. Stowe's theorem requires that $H^{1}\left(\Gamma(x) ; T_{x} X\right)=0$ where the coefficients are a $\Gamma(x)$-module via derivative action at $x$. We also require that the local isotropy representation on $T_{x} X$ be rigid, which by Weil's Theorem $[65,66,67]$ follows from the vanishing of the first cohomology for the adjoint representation on $\mathbf{g l}\left(T_{x} X\right)$. Both of these conditions are implied by: 
DEFINITION 2.1 $\Gamma$ satisfies a strong vanishing cohomology condition if:

$\mathbf{S V C}(\mathbf{N}) \quad H^{1}\left(\tilde{\Gamma} ; \mathbf{R}_{\tilde{\rho}}^{N}\right)=\{0\}$ for every subgroup $\tilde{\Gamma} \subset \Gamma$ of finite index and representation $\tilde{\rho}: \tilde{\Gamma} \rightarrow G L(N, \mathbf{R})$.

Observe that if $\Gamma$ satisfies $\operatorname{SVC}(\mathrm{N})$, then every subgroup $\tilde{\Gamma} \subset \Gamma$ of finite index also satisfies $\mathrm{SVC}(\mathrm{N})$. By a very remarkable result of $\mathrm{G}$. A. Margulis, condition $\mathrm{SVC}(\mathrm{N})$ for arbitrary $\mathrm{N}$ holds for any subgroup $\Gamma$ of finite-index in $S L(n, \mathbf{Z})$ for $n \geq 3$, as well as for many other lattices in higher-rank semi-simple Lie groups (cf. Theorem 2.1, [49]):

THEOREM 2.2 (Margulis) Let $\Gamma \subset G$ be an irreducible lattice in a connected semi-simple algebraic $\mathbf{R}$-group $G$. Assume that the $\mathbf{R}$-split rank of each factor of $G$ is at least 2, and that $G_{\mathbf{R}}^{0}$ has no compact factors. Then $\Gamma$ satisfies condition $S V C(N)$ for every $N>0$.

The Kunneth formula in cohomology implies that a product of groups satisfying condition $\mathrm{SVC}(\mathrm{N})$ will also satisfy $\mathrm{SVC}(\mathrm{N})$, so that Margulis' Theorem implies that SVC(N) holds for products of lattices as in Theorem 2.2.

A $C^{0, r}$-deformation of an action $\varphi$ is a continuous path of $C^{r}$-actions $\varphi_{t}$ defined for some $0 \leq t \leq \epsilon$ with $\varphi_{0}=\varphi$. An action $\varphi$ is said to be topologically deformation rigid if every $C^{0,1}$-deformation of $\varphi$, with $\varphi_{t}$ contained in a sufficiently small $C^{1}$-neighborhood of $\varphi$, is topologically conjugate to $\varphi$ by a continuous path of homeomorphisms $\left\{H_{t} \mid 0 \leq t \leq \epsilon\right\}$. That is, for each $\gamma \in \Gamma$ and $0 \leq t \leq \epsilon$ we have

$$
\begin{aligned}
H_{t}^{-1} \circ \varphi_{t}(\gamma) \circ H_{t} & =\varphi(\gamma) \\
H_{0} & =I d_{X}
\end{aligned}
$$

The approach to geometric rigidity for Anosov actions is based on ideas from dynamical systems, and especially follows the philosophy that an Anosov dynamical system is determined by its behavior at periodic orbits. The Anosov hypothesis is used to reduce proofs to questions about the behavior at periodic orbits. This is a standard method for the study of Anosov diffeomorphisms (cf. especially [2, 38, 18, 43, 31, 58, 59].)

The author's approach to topological deformation rigidity studies the behavior of the periodic orbits for the system under deformation, following the above philosophy. The Anosov hypothesis guarantees that these periodic orbits are always isolated, with a unique fixed-point for their associated linear isotropy actions. The hypotheses condition $\mathrm{SVC}(\mathrm{N})$ on the first cohomology of the group $\Gamma$ implies, by the stability theorem of D. Stowe [60, 61], that the periodic orbits are "uniformly" continuously stable under a $C^{0,1}$-deformation of an action. The existence of a $\varphi$-hyperbolic element $\gamma_{h}$ implies there is a unique candidate family of homeomorphisms which satisfy (1), the path which works for $\varphi_{t}\left(\gamma_{h}\right)$ by Anosov structural stability. The stability of the fixed-points implies this path works for the whole group, which yields the first result: 
THEOREM 2.3 (Theorem 2.9 [28]) Let $\varphi: \Gamma \times X \rightarrow X$ be an Anosov $C^{1}$ action on a compact $n$-manifold $X$ such that the periodic points $\Lambda(\varphi)$ are dense in $X$, and $\Gamma$ has the $S V C(n)$. Then $\varphi$ is topologically deformation rigid.

The conclusion of Theorem 2.3 is false for the standard action of $S L(2, \mathbf{Z})$ on the 2-torus: Example 7 below shows that the standard action can be smoothly deformed through volume-preserving $C^{\omega}$-actions which are not topologically conjugate to the standard linear action.

A $C^{r}$-action $\varphi_{1}$ is said to be a $C^{1}$-perturbation of a $\mathrm{C}^{r}$-action $\varphi$, for a fixed finite set of generators $\left\{\delta_{1}, \ldots, \delta_{d}\right\}$ of $\Gamma$, if the $C^{r}$-diffeomorphisms $\varphi\left(\delta_{i}\right)$ and $\varphi_{1}\left(\delta_{i}\right)$ are $C^{1}$-close for all $i$. The action $\varphi$ is said to be $C^{r}$-rigid (or topologically rigid if $r=0$ ) if every sufficiently small $C^{1}$-perturbation of $\varphi$ is $C^{r}$-conjugate to $\varphi$, for $0 \leq r \leq \infty$, or $r=\omega$ in the case of real analytic actions. That is, there is a $C^{r}$-diffeomorphism, $H_{1}: X \rightarrow X$, such that for each $\gamma \in \Gamma$ we have

$$
H_{1}^{-1} \circ \varphi_{1}(\gamma) \circ H_{1}=\varphi(\gamma)
$$

For a $C^{1}$-perturbation of an Anosov action, if we fix a $\varphi$-hyperbolic element $\gamma_{h} \in \Gamma$, then there is a unique candidate $H_{1}$ (in the isotopy class of the identity) for the solution to (2), obtained from Anosov structural stability for $\varphi\left(\gamma_{h}\right)$. In fact, the group $\Gamma$ is easily seen to be generated by $\varphi$-hyperbolic elements $\left\{\gamma_{1}, \ldots, \gamma_{d}\right\}$, so topological stability is equivalent to showing that there is one homeomorphism $H_{1}$ which works for all of the $\gamma_{i}$. Theorem 2.3 establishes deformation rigidity by noting that all of these conjugacies agree for the initial action (as they are then all the identity!) and for $t>0$, Stowe's Theorem and connectedness of the parameter path implies they must agree on the dense set of periodic points $\Lambda\left(\varphi_{t}\right)$. By continuity of the homeomorphisms $\left\{H_{t}\right\}$, they must agree everywhere. For a $C^{1}$-perturbation $\varphi_{1}$ which is not connected to $\varphi$ by a $C^{0,1}$-path of actions, this method yields control over only a finite subset of $\Lambda\left(\varphi_{1}\right)$, so that topological rigidity is not known in the generality of Theorem 2.3 .

A. Katok and J. Lewis introduced a new technique in [34] for the study of the topological rigidity of $S L(n, \mathbf{Z})$. Their key observation was that for $n \geq 4$, there are many embeddings of the non-trivial product groups $S L\left(n_{1}\right) \times S L\left(n_{2}\right) \subset$ $S L(n, \mathbf{Z})$ where $n_{1}+n_{2}=n$, whose actions on $\mathbf{T}^{n}$ are normally hyperbolic along a dense set of invariant tori of lower dimensions. One can apply Stowe's theory for these subgroups to obtain perturbation stability for a family of sub-tori in $\mathbf{T}^{n}$. This additional information suffices to yield "dynamical control" of all points in $\Lambda\left(\varphi_{1}\right)$ for a $C^{1}$-perturbation. That is, the various choices of topological conjugacies for a hyperbolic generating set of $S L(n, \mathbf{Z})$ can be shown to agree on the periodic points, so that they obtained:

THEOREM 2.4 (Theorem 1.3 [34]) Let $\Gamma \subset S L(n, \mathbf{Z})$ be a subgroup of finite index for $n \geq 4$. Then the standard action of $\Gamma$ on $\mathbf{T}^{n}$ is topologically rigid.

The method of Katok and Lewis also extends to cover case of the integer symplectic matrices $S p(n, \mathbf{Z}) \subset S L(2 n, \mathbf{Z})$ and products of the two types of actions: 
THEOREM 2.5 (Theorem 6.1 [34]) Let $\Gamma \subset S p(2 n, \mathbf{Z})$ be a subgroup of finite index for $n \geq 3$. Then the standard action of $\Gamma$ on $\mathbf{T}^{2 n}$ is topologically rigid.

THEOREM 2.6 (Theorem 6.2 [34]) Let $\Gamma_{i}$ be a subgroup of finite index in $S L\left(n_{i}, \mathbf{Z}\right)$ for $n_{i} \geq 4$, or $S p\left(n_{i}, \mathbf{Z}\right)$ for $n_{i} \geq 3$, and set $m_{i}=n_{i}$ or $2 n_{i}$, respectively. Then the standard action of $\Gamma_{1} \times \cdots \times \Gamma_{k}$ on $\mathbf{T}^{n}=\mathbf{T}^{m_{1}} \times \mathbf{T}^{m_{k}}$ is topologically rigid.

Note that Theorem 2.3 yields topological deformation rigidity for Anosov actions on examples including tori and infra-nilmanifolds, while the methods of Katok and Lewis yield topological rigidity for certain examples of standard actions on tori. Extending topological rigidity to other subgroups of $S L(n, \mathbf{Z})$ and to actions on general nilmanifolds is a central problem. The task appears to be non-trivial, for one is looking for a dynamical replacement of the use of the concrete algebraic structures used by Katok and Lewis to control the periodic structures, both points and submanifolds, for perturbations of Anosov actions of large groups. For example, in the passage from the full integer matrix group to a subgroup of finite index, the Katok and Lewis method invokes the solution of the congruence subgroup problem $[50,3,4]$, so that a dynamical theory which avoids this algebraic result is likely to have great depth.

Recently, Mark Pollicott has developed criteria for a standard Anosov action to have simultaneous structural stability homeomorphisms (conjugating the generators of a $C^{1}$-close action to the standard generators). His method replaces control of the conjugating homeomorphisms with a global fixed-point theorem for the sections of a Banach bundle, where the vanishing cohomology condition can be viewed as having coefficients in a Banach space. This is a direct adaptation of Moser's proof of Anosov $C^{1}$-stability to group actions, yielding in particular:

THEOREM 2.7 (Theorem [54]) The standard action $A: S L(n, \mathbf{Z}) \times \mathbf{T}^{n} \rightarrow$ $\mathbf{T}^{n}$ for $n \geq 3$ is locally topologically rigid.

Pollicott's method produces a parameter space for the set of nearby actions modulo conjugacy, which he shows to be trivial for the groups $S L(n, \mathbf{Z})$. This approach is attractive for its directness, and also because it is applicable to actions where rigidity is not expected. In the latter situation, the problem of classification becomes a question of understanding the parameter space of all actions modulo conjugacy, which is an absolutely open problem. (cf. Theorem 6.1 below for an example of an action with a non-trivial parameter space.)

The above results are concerned with showing that a $C^{1}$-perturbation of an Anosov action is topologically standard. One can also ask the more ambitious question: what are the hypotheses on an Anosov action which yields global algebraic rigidity? Katok and Lewis have extended their perturbation techniques to obtain just such a result: 
THEOREM 2.8 (Theorem 2.1 [33]) Suppose that $\Gamma$ is a subgroup of finite index in $S L(n, \mathbf{Z}), n \geq 4$, and $\varphi: \Gamma \times \mathbf{T}^{n} \rightarrow \mathbf{T}^{n}$ is a $C^{1}$-action so that:

1. there exists a fixed-point for the action; i.e., there exists $x_{0} \in \mathbf{T}^{n}$ such that $\varphi(\gamma)\left(x_{0}\right)=x_{0}$ for every $\gamma \in \Gamma$.

2. There exists a direct-sum decomposition of $\mathbf{Q}^{n}$ as a vector space over $\mathbf{Q}$,

$$
\mathbf{Q}^{n}=\mathbf{V}_{1} \oplus \mathbf{V}_{2}, \quad \mathbf{V}_{1} \cong \mathbf{Q}^{k}, \quad \mathbf{V}_{2} \cong \mathbf{Q}^{\ell}, k+\ell=n, k, \ell \geq 2
$$

and a $\varphi$-hyperbolic element $\gamma_{h} \in \Gamma$ such that $\varphi\left(\gamma_{h}\right)\left(\mathbf{V}_{i}\right)=\mathbf{V}_{i}$ for $i=1,2$.

Then $\varphi$ is topologically conjugate to the standard linear action on $\mathbf{T}^{n}$ induced from the action of $\varphi^{*}$ on $H^{1}\left(\mathbf{T}^{n} ; \mathbf{R}\right)$.

The other known global rigidity results all follow from the methods of cocycle super-rigidity, which is discussed in section 5 below.

\section{From topological to smooth rigidity}

The central problem for Anosov dynamical systems with one generator is to find conditions under which topological conjugacy implies smooth conjugacy $[5,8,19,20,31,40,41,42,44,43,47,48,53,52]$. For a volume-preserving Anosov $C^{r}$-diffeomorphism of $\mathbf{T}^{2}$ for $1 \leq r \leq \omega$, R. de la Llavé showed in $[40,41]$ that the exponents in the stable and unstable directions at periodic points form a complete set of smooth invariants of conjugacy. That is, if $H$ is a topological conjugacy between two volume-preserving Anosov actions $\phi_{0}$ and $\phi_{1}$, such that the exponents of $D \phi_{0}$ at every periodic point $x \in \mathbf{T}^{2}$ agree with those of $D \phi_{1}$ at $H(x)$, then $H$ is a $C^{r}$-diffeomorphism. The key to this result is the Livsic Theorem referred to previously, which along with the hyperbolicity of the action is used to prove that $H$ must be $C^{r}$ when restricted to the 1dimensional stable and unstable leaves of $\phi_{0}$. Remarkably, this implies the map $H$ is $C^{r}$ when $r=\infty$ or $\omega$, and for $r>1$ the map $H$ is $C^{r-\epsilon}$ for some $\epsilon>0$ independent of $r$. (See Lemma 2.3 [43].) This method uses in a key way that the stable and unstable foliations are 1-dimensional, and are conjugate under the homeomorphism $H$.

The interesting actions of lattices on compact manifolds, for which rigidity can be expected, all require the dimension $n \geq 3$. One of the points of the works $[25,28]$ was that the method of de la Llavé can be made to work in higher dimensions as well, by taking advantage of the extra group structure present for a higher rank lattice. This leads to the notion of a trellised action, Definition 3.2. Briefly, this is an Anosov action with sufficiently many hyperbolic elements which preserve a maximally transverse system of "sufficiently regular" 1-dimensional foliations of $X$. These foliations then yield a dynamically defined affine structure on $X$ which is stable under topological conjugation.

The problem of showing that a topological conjugacy between Anosov actions of a higher rank lattice must be smooth, hinges on a much simpler problem, whether a topological conjugacy between two Anosov actions of an abelian 
group $\mathcal{A}$ on $X$ must be smooth. This very interesting question is addressed later in this section, including the regularity results of the author [28] and of Katok and Lewis [34] that are part of the classification of lattice actions, and the more recent work of Katok and Spatzier [36] on regularity of abelian actions.

We begin with the definition of a trellis structure:

DEFINITION 3.1 Let $X$ be a compact smooth $n$-manifold without boundary. Let $1 \leq r \leq \infty$, or $r=\omega$ for the real analytic case. $A C^{r}$-trellis $\mathcal{T}$ on $X$ is a collection of 1-dimensional, pairwise-transverse foliations $\left\{\mathcal{F}_{i} \mid 1 \leq i \leq n\right\}$ of $X$ such that

1. The tangential distributions have internal direct sum $T \mathcal{F}_{1} \oplus \cdots \oplus T \mathcal{F}_{n} \cong$ $T X$

2. For each $x \in X$ and $1 \leq i \leq n$, the leaf $L_{i}(x)$ of $\mathcal{F}_{i}$ through $x$ is a $C^{r}$ immersed submanifold of $X$;

3. The $C^{r}$-immersions $L_{i}(x) \hookrightarrow X$ depend uniformly Hölder continuously on the basepoint $x$ in the $C^{r}$-topology on immersions.

$\mathcal{T}$ is a regular $C^{r}$-trellis if it also satisfies the additional condition:

4. Each foliation $\mathcal{F}_{i}$ is transversally absolutely continuous with a quasi-invariant transverse volume form that depends smoothly on the leaf coordinates.

All methods of proving regularity of a topological conjugacy between Anosov actions seem to require that the given group action "preserve" a trellis on $X$, which we formulate as:

DEFINITION 3.2 (Trellised Action) $A C^{r}$-action $\varphi: \Gamma \times X^{n} \rightarrow X^{n}$ is trellised if there exist:

1. a regular $C^{r}$-trellis $\mathcal{T}=\left\{\mathcal{F}_{i} \mid 1 \leq i \leq n\right\}$ on $X$;

2. $\varphi$-hyperbolic elements $\Delta=\left\{\gamma_{1}, \ldots, \gamma_{n}\right\} \subset \Gamma$ such that $\mathcal{F}_{i}$ is invariant under the Anosov diffeomorphism $\varphi\left(\gamma_{i}\right)$. That is, $\varphi\left(\gamma_{i}\right)$ maps each leaf of $\mathcal{F}_{i}$ to a leaf of $\mathcal{F}_{i}$.

$\varphi$ is an oriented trellised action if (3.2.1) and (3.2.2) hold, and in addition:

3. each of the tangential distributions $T \mathcal{F}_{i}$ is oriented and the Anosov diffeomorphism $\varphi\left(\gamma_{i}\right)$ preserves the orientation of $T \mathcal{F}_{i}$.

$\varphi$ is a volume-preserving trellised action if (3.2.1) and (3.2.2) hold, and in addition:

4. there is a $C^{r}$-volume-form on $X$ which is invariant under the action of the $\varphi$-hyperbolic elements $\gamma_{i} \in \Delta$.

The elements $\gamma_{i}$ are not required to commute in the definition of a trellised action. Moreover, we do not require that $\mathcal{F}_{i}$ be the stable, or even the strongest stable foliation of $\varphi\left(\gamma_{i}\right)$. The present definition allows, for example, that there is one fixed $\gamma \in \Gamma$ such that $\gamma_{i}=\gamma$ for all $1 \leq i \leq n$; such a $\gamma$ would then be a "dynamical" regular semi-simple element for $\Gamma$.

The author proved the following regularity result for deformations of a trellised action: 
THEOREM 3.3 (Theorem 2.12 [28]) For a closed $n$-manifold $X$, suppose that:

1. $\varphi_{0}: \Gamma \times X \rightarrow X$ is a $C^{r}$-action with dense periodic orbits, for $r=1, \infty$ or $\omega ;$

2. $\Gamma$ is finitely-generated, and satisfies the cohomology condition $S V C\left(n^{2}-1\right)$;

3. $\varphi_{0}$ is trellised by a regular trellis $\mathcal{T}_{0}$, with associated hyperbolic elements $\Delta=\left\{\gamma_{1}, \ldots, \gamma_{n}\right\}$

4. $\left\{\varphi_{t} \mid 0 \leq t \leq \epsilon\right\}$ is a $C^{0, r}$-deformation of $\varphi_{0}$ such that $\varphi_{t}\left(\gamma_{i}\right)$ is Anosov for all $1 \leq i \leq n$ and $0 \leq t \leq \epsilon$;

5. $\varphi_{t}$ is conjugate to $\varphi_{0}$ by a continuous family of homeomorphisms $\left\{H_{t}: X \rightarrow X \mid 0 \leq t \leq \epsilon\right\}$

6. there is a $C^{r}$-trellis $\mathcal{T}_{t}$ on $X$ such that $H_{t}$ maps the leaves of $\mathcal{F}_{0, i}$ to those of $\mathcal{F}_{t, i}$.

Then $H_{t}$ is a $C^{r}$-diffeomorphism for all $0 \leq t \leq \epsilon$.

Suppose, in addition, that $\left\{\varphi_{t} \mid 0 \leq t \leq \epsilon\right\}$ is a $C^{0, \ell}$-deformation for $\ell=1$, or $\ell=\infty$ if $r=\infty$ or $\omega$, and the leaves of the foliations $\left\{\mathcal{F}_{t, i}\right\}$ depend continuously on the parametert in the $C^{\ell}$-topology on immersions. Then the diffeomorphisms $H_{t}$ depend continuously on $t$ in the $C^{\ell}$-topology on maps.

This theorem is technical in its assumptions, and to obtain its hypotheses in applications it is necessary to impose further assumptions on $\Gamma$ and the initial action $\varphi_{0}$. For example, the class of Cartan actions introduced in [28] are always trellised. First, let us recall a special property of hyperbolic elements, which corresponds in the linear case to a matrix $\gamma \in S L(n, \mathbf{Z})$ having a unique maximally contracting direction:

DEFINITION 3.4 An Anosov map $f$ has a one-dimensional strongest stable distribution if there exists a Df-invariant, 1-dimensional vector subbundle $E^{s s} \subset E^{-}$which satisfies an exponential dichotomy: that is, there exists

- a Finsler on TX,

- a continuous splitting of the tangent bundle into Df-invariant subbundles, $T X \cong E^{c s} \oplus E^{s s}$,

- constants $\lambda>1$ and $1>\epsilon>0$

such that for all positive integers $m$,

$$
\begin{aligned}
& \left\|D\left(f^{m}\right)(v)\right\|>(\lambda-\epsilon)^{-m} \cdot\|v\| ; \quad 0 \neq v \in E^{c s} \\
& \left\|D\left(f^{m}\right)(v)\right\|<(\lambda+\epsilon)^{-m} \cdot\|v\| ; \quad 0 \neq v \in E^{s s} .
\end{aligned}
$$

The strongest stable distribution $E^{s s}$ is necessarily integrable, and the leaves of the resulting foliation $\mathcal{F}^{s s}$ are $C^{r}$-immersed 1-dimensional submanifolds (cf. Chapter 6 [58]). While the foliation $\mathcal{F}^{s s}$ need not even be $C^{1}$, the immersions of its leaves vary Hölder continuously in the $C^{r}$-topology on immersions into $X$. 
DEFINITION 3.5 (Abelian Cartan Action) Let $\mathcal{A}$ be a free abelian group with a given set of generators $\Delta=\left\{\gamma_{1}, \ldots, \gamma_{n}\right\} . \quad(\varphi, \Delta)$ is a Cartan $C^{r}$-action on the n-manifold $X$ if:

- $\varphi: \mathcal{A} \times X \rightarrow X$ a $C^{r}$-action on $X$;

- each $\gamma_{i} \in \Delta$ is $\varphi$-hyperbolic and $\varphi\left(\gamma_{i}\right)$ has a 1-dimensional strongest stable foliation $\mathcal{F}_{i}^{\text {ss }}$

- the tangential distributions $E_{i}^{s s}=T \mathcal{F}_{i}^{s s}$ are pairwise-transverse with their internal direct sum $E_{1}^{s s} \oplus \cdots \oplus E_{n}^{s s} \cong T X$.

We say that $(\varphi, \Delta)$ is a maximal Cartan action if $\varphi$ is a Cartan action, and for each $1 \leq i \leq n$, the stable foliation $\mathcal{F}_{i}$ of the Anosov diffeomorphism $\varphi\left(\gamma_{i}\right)$ is 1-dimensional; hence $\mathcal{F}_{i}=\mathcal{F}_{i}^{s s}$.

We say that $(\varphi, \Delta)$ is an orientable Cartan action if each trellis foliation $\mathcal{F}_{i}$ is orientable, and the restricted action of each $\varphi(\gamma)$ on $\mathcal{F}_{i}$ for $\gamma \in \Delta$ is orientation-preserving.

\section{THEOREM 3.6 (Theorem $2.16[28]$ )}

1. For a Cartan $C^{r}$-action $(\varphi, \Delta)$, the collection of strongest stable foliations $\mathcal{T}=\left\{\mathcal{F}_{1}^{s s}, \ldots, \mathcal{F}_{n}^{s s}\right\}$ is a $C^{r}$-trellis on $X$.

2. For a maximal Cartan $C^{r}$-action $(\varphi, \Delta)$, the collection of stable foliations $\mathcal{T}=\left\{\mathcal{F}_{1}, \ldots, \mathcal{F}_{n}\right\}$ is a regular $C^{r}$-trellis on $X$.

3. For a volume-preserving maximal Cartan $C^{r}$-action $(\varphi, \Delta)$ with $r \geq 3$, each stable foliation $\mathcal{F}_{i}$ is transversally $C^{1+\alpha}$ for some $0<\alpha<1$.

If we assume that $X$ is a compact nilmanifold, then the trellis associated to the generators of a Cartan action are topological invariants:

THEOREM 3.7 (Theorem 1.1 [30]) Let $\mathcal{A}$ be a free abelian group, and $\left(\varphi_{0}, \Delta\right)$ and $\left(\varphi_{1}, \Delta\right)$ be two Cartan $C^{1}$-actions on a compact nilmanifold $X$. Suppose that there exists a homeomorphism $H: X \rightarrow X$ conjugating the action of $\varphi_{1}$ to $\varphi_{0}$. Then for each $1 \leq i \leq n, H$ maps the strongest stable foliation of $\varphi_{1}\left(\gamma_{i}\right)$ to that of $\varphi_{0}\left(\gamma_{i}\right)$.

It is an elementary consequence of the work work of Franks [15] and Newhouse [51] that a transitive Anosov action of an abelian group on a torus with a common fixed-point is topologically equivalent to an algebraic action. This result is also true for Anosov actions on infra-nilmanifolds using the corresponding work of Manning [46]:

THEOREM 3.8 (Proposition $2.18[\mathbf{2 8}]$ ) Let $(\varphi, \Delta)$ be a Cartan $C^{1}$-action on the closed $n$-dimensional infra-nilmanifold $X$. Then $\varphi$ has a periodic point $x_{0}$, and there is a positive integer $p$ so that the action of the $p^{\text {th }}$-powers $\Delta^{p}=$ $\left\{\gamma_{1}^{p}, \ldots, \gamma_{m}^{p}\right\}$ is topologically conjugate to a standard (algebraic) Cartan action induced by the map on homotopy, $\varphi_{\#}: \Delta^{p} \times \pi_{1}\left(X ; x_{0}\right) \rightarrow \pi_{1}\left(X ; x_{0}\right)$. 
The author has produced examples of abelian Cartan actions which do not have a fixed-point for the full action [27], so that the reduction to the subgroup generated by $\Delta^{p}$ is necessary.

We have described the known topological properties of abelian Cartan actions. Let us now address the question of their smooth rigidity. Theorem 3.8 reduces the problem to showing that a topological conjugacy between abelian Cartan actions, which by Theorem 3.7 preserves the trellising, is smooth when restricted to the leaves of the trellis foliations. The techniques of de la Llavé then directly carry over to show the conjugacy is as smooth as the actions (cf. section 5, [28].) There are two methods currently known which are sufficient to guarantee that a conjugacy is smooth when restricted to the strongest stable leaves:

- Assume that the exponents of the action match up at periodic orbits, exactly as in the case of $\mathbf{T}^{2}$;

- Assume that the dynamics of the Cartan action matches up the unique Gibbs measures along the strongest stable foliations.

The former approach is taken in [25, 28], the latter in $[8,34,42]$. We state some results that follow from each of these hypotheses. Theorems 3.9 and 3.11 below generalize to higher dimensions results of R. de la Llavé, J. Marco and R. Moriyon $[40,41,44,43,47,48]$ for $X=\mathbf{T}^{2}$.

THEOREM 3.9 (Theorem 2.19 [28]) Let $\mathcal{A}$ be an abelian group generated, not necessarily freely, by the set $\Delta=\left\{\gamma_{1}, \ldots, \gamma_{n}\right\}$. Given volume-preserving Cartan $C^{r}$-actions $\left(\varphi_{0}, \Delta\right)$ and $\left(\varphi_{1}, \Delta\right)$ on an $n$-manifold $X$, for $r=1, \infty$ or $\omega$, suppose that:

1. $\varphi_{0}$ is a trellised action (i.e., the foliations have additional regularity);

2. $H: X \rightarrow X$ is a homeomorphism conjugating $\varphi_{1}$ to $\varphi_{0}$;

3. For all $1 \leq i \leq n$ and for each $x \in \Lambda\left(\varphi_{0}\right)$, the maximally contracting exponent of $D_{x} \varphi_{0}\left(\gamma_{i}\right)$ equals the maximally contracting exponent of $D_{H(x)} \varphi_{1}\left(\gamma_{i}\right)$.

Then $H$ is a $C^{r}$-diffeomorphism. Moreover, for $\ell=1$ (or $\ell=\infty$ if $r=\infty$ or $\omega)$ suppose there are given

4. a $C^{0, \ell}$-deformation $\left\{\left(\varphi_{t}, \Delta\right) \mid 0 \leq t \leq 1\right\}$ through volume-preserving Cartan $C^{r}$-actions, and

5. a continuous family of homeomorphisms $\left\{H_{t} \mid 0 \leq t \leq 1\right\}$ conjugating $\varphi_{t}$ to $\varphi_{0}$

which satisfy (3.9.2) and (3.9.3) for all $0 \leq t \leq 1$. Then the $C^{r}$-diffeomorphism $H_{t}$ depends $C^{0}$ on $t$ in the $C^{\ell}$-topology on $C^{r}$-maps.

COROLLARY 3.10 (Corollary 2.20 [28]) Let $\left(\varphi_{0}, \Delta\right)$ be a volume-preserving trellised Cartan $C^{r}$-action on an n-manifold $X$, for $r=\infty$ or $\omega$, with $\mathcal{A}$ the abelian group generated, not necessarily freely, by the set $\Delta=\left\{\gamma_{1}, \ldots, \gamma_{n}\right\}$. Suppose that $H: X \rightarrow X$ is a $C^{1}$-conjugacy between an arbitrary $C^{r}$-action 
$\varphi_{1}: \mathcal{A} \times X \rightarrow X$ and the given action $\varphi_{0}$. Then $\left(\varphi_{1}, \Delta\right)$ is a volume-preserving Cartan $C^{r}$-action, and $H$ is a $C^{r}$-diffeomorphism.

For a Cartan action $\varphi$, let $x \in \Lambda$ be a periodic point, and let $\mathcal{A}_{x}$ be the isotropy subgroup of $x$. The linear isotropy representation

$$
D_{x} \varphi: \mathcal{A}_{x} \rightarrow G L\left(T_{x} X\right)
$$

has image in a maximal diagonal subgroup. The choice of a trellis $\left\{\mathcal{F}_{i}\right\}$ for the action defines a basis in each tangent space $T_{x} X$ for which the action is diagonal. Introduce the abelian (multiplicative) diagonal group $\mathbf{R}^{+} \oplus \cdots \oplus \mathbf{R}^{+}$, then we can consider the isotropy representations as homomorphisms $D_{x} \varphi: \mathcal{A}_{x} \rightarrow \lambda^{n}$. An orientable Cartan action is said to have constant exponents if there exist homomorphisms $\lambda_{i}: \mathcal{A} \rightarrow \mathbf{R}^{+}$for $1 \leq i \leq n$ such that for each $x \in \Lambda$ and $\gamma \in \mathcal{A}_{x}$

$$
D_{x} \varphi(\gamma)=\lambda_{1}(\gamma) \oplus \ldots \oplus \lambda_{n}(\gamma)
$$

THEOREM 3.11 (Theorem 2.21 [28], Theorem $1.2[30]$ ) Let $(\varphi, \Delta)$ be a Cartan $C^{r}$-action on an infra-nilmanifold $X$, for $r=1, \infty$ or $\omega$. If $\varphi$ has constant exponents, then there is subgroup $\tilde{\mathcal{A}} \subset \mathcal{A}$ of finite-index so that the restriction $\varphi \mid \tilde{\mathcal{A}}$ is $C^{r}$-conjugate to a standard linear action, and $\varphi$ is $C^{r}$-conjugate to an affine action of $\mathcal{A}$ on $X$.

Katok and Lewis developed a form of "non-stationary Sternberg linearization" in the Appendix to [34] which extended the work of B. Anderson (Lemma, page $145[1]$ ), and used this to prove for a maximal Cartan action:

THEOREM 3.12 (Theorems 4.2 and $4.12[34]$ ) Let $\varphi: \mathcal{A} \times \mathbf{T}^{n} \rightarrow \mathbf{T}^{n}$ be a maximal Cartan $C^{\infty}$-action for $n \geq 3$ with a fixed-point $x_{0}$ for the action. Then the Franks-Newhouse topological conjugacy $H: \mathbf{T}^{n} \rightarrow \mathbf{T}^{n}$ between $\varphi$ and the standard action $\varphi_{*}: \mathcal{A} \times \mathbf{T}^{n} \rightarrow \mathbf{T}^{n}$ is $C^{\infty}$.

Finally, we return to the main issue, which is to show that a topological conjugacy between Anosov actions of higher rank lattices is necessarily smooth. From the previous discussion, it is clear that we would like to reduce the question to either of the cases Theorem 3.9 or Theorem 3.12. The idea introduced in $[25,28]$ was to look for an abelian subgroup $\mathcal{A}$ of the given lattice $\Gamma$, then restrict the action to $\mathcal{A}$ and require that the restriction be Cartan. The existence of a free abelian subgroup $\mathcal{A}$ of rank $n-1$, for any $\Gamma \subset S L(n, \mathbf{Z})$ of finite index, can be shown by number-theoretic methods. However, there is a much more powerful existence theorem which applies to every subgroup $\Gamma \subset G_{\mathbf{Z}}$ of finite index, due to G. Prasad and M. S. Raghunathan:

THEOREM 3.13 (Theorem 2.8 and Corollary 2.9 [55]) Let $G$ be a semi-simple analytic Lie group and $\Gamma$ a lattice in $G$. Let $H$ be a Cartan subgroup of $G$, then there exists $g \in G$ such that $\Gamma_{H}=\Gamma \cap g^{-1} H g$ is a uniform lattice in $g^{-1} \mathrm{Hg}$. 
If $G$ is a semi-simple linear group with no compact factors, then we can apply Theorem 3.13 for $H$ a maximal $\mathbf{R}$-split torus to conclude that a lattice $\Gamma$ in $G$ always contains a free abelian subgroup $\mathcal{A}$ of rank equal to the rank of $G$, such that the generators of $\mathcal{A}$ are represented by commuting, diagonalizable hyperbolic elements. We call the resulting subgroup $\Gamma_{H}$ a Cartan subgroup for $\Gamma$. The standard action of $\Gamma_{H}$ on $\mathbf{T}^{N}$ will be a Cartan action, if the Lie group $H$ can be simultaneously diagonalized with by a basis $\left\{v_{1}, \ldots, v_{N}\right\}$ of $\mathbf{R}^{N}$ so that each $v_{i}$ is the (unique up to scalar multiples) maximal eigenvector for some $g_{i} \in H$. This is a Lie algebraic question which can be easily determined in all examples.

The following two results on the smoothness of a topological conjugacy between trellised Cartan actions use the strategy of controlling the exponents at periodic orbits:

THEOREM 3.14 (Theorem 2.15 [28]) For a closed $n$-manifold $X$, suppose that:

1. $\varphi_{0}: \Gamma \times X \rightarrow X$ is a $C^{r}$-action of $\Gamma$ with dense periodic orbits, for $r=1, \infty$ or $\omega$;

2. $\varphi_{0}$ is trellised by a regular trellis $\mathcal{T}_{0}$ whose associated hyperbolic elements $\Delta=\left\{\gamma_{1}, \ldots, \gamma_{n}\right\}$ determine an abelian Cartan subaction $\varphi_{0} \mid \mathcal{A} ;$

3. $\Gamma$ is a higher-rank lattice in a Lie group $G$, and the subgroup $\mathcal{A} \subset \Gamma$ generated by $\Delta$ is a cocompact lattice in a maximal $\mathbf{R}$-split torus of $G$;

4. $\varphi_{1}: \Gamma \times X \rightarrow X$ is a $C^{r}$-action such that $\varphi_{1} \mid \mathcal{A}$ is an abelian Cartan subaction;

5. $\varphi_{1}$ is conjugate to $\varphi_{0}$ by a homeomorphism, $H: X \rightarrow X$

Then $H$ is a $C^{r}$-diffeomorphism.

Katok and Lewis obtained the following global result, which is remarkable in that only the Anosov hypothesis is made on the dynamics of the action $\varphi$ ! Note that it does not even assume that the given action preserves a volume form; this is deduced from the dynamics and the given topological conjugacy to a standard action.

THEOREM 3.15 Let $\Gamma$ be a subgroup of finite index in $S L(n, \mathbf{Z}), n \geq 3$, and $\varphi: \Gamma \times \mathbf{T}^{n} \rightarrow \mathbf{T}^{n}$ an Anosov $C^{\infty}$-action. Suppose that $H: \mathbf{T}^{n} \rightarrow \mathbf{T}^{n}$ is a topological conjugacy between $\varphi$ and the standard action $\varphi^{*}$ induced on $\mathbf{T}^{n} \cong H^{1}\left(\mathbf{T}^{n} ; \mathbf{R}\right) / H^{1}\left(\mathbf{T}^{n} ; \mathbf{Z}\right)$. Then $H$ is necessarily $C^{\infty}$.

This theorem is typical of the best result one could hope for in the subject. We do not go into the discussion of the proof, but mention that a key rôle is played by the ideas of cocycle super-rigidity, which are used to show that the exponents for an abelian Cartan subaction agree at periodic points. The mention of super-rigidity brings us to the last phase of the rigidity theory, which are the global results obtained from combining extensions of super-rigidity with Theorem 3.11. 


\section{Smooth rigidity for Cartan actions}

The original "global rigidity result" for volume-preserving smooth ergodic actions of higher rank lattices on a compact manifold is the cocycle super-rigidity theorem of R. Zimmer [68, 70]: with appropriate technical hypotheses on the action $\varphi: \Gamma \times X \rightarrow X$, there is a measurable framing of the tangent bundle $T X$ so that with respect to this framing the matrix-valued derivative cocycle $D \varphi: \Gamma \times X \rightarrow G L(n, \mathbf{R})$ is almost everywhere given by a homomorphism $\rho: \Gamma \rightarrow G L(n, \mathbf{R})$. In a sense, this says that the action of almost-everywhere infinitesimally standard.

On the other hand, the study of abelian Cartan $C^{r}$-actions shows that the exponents of the derivative mapping at periodic orbits for the action form a set of moduli for their smooth classification. In particular, if the exponents are constant at every periodic orbit, then Theorem 3.11 states that the Cartan action is affine, and hence there is a finite-index subgroup whose action is standard. The condition on the constancy of the exponents is equivalent to a type of super-rigidity conclusion which is valid on the dense set of periodic points.

At a conference on "Geometric Rigidity" at Penn State in March 1991, the talks by J. Lewis and R. Zimmer discussed an application of super-rigidity to the classification of actions of finite-index subgroups of $S L(n, \mathbf{Z})$ on $\mathbf{T}^{n}$. The conclusion of super-rigidity was shown to apply for these groups, with an additional hypotheses of an invariant volume form. Lewis and Zimmer then used Theorem 3.12 to deduce a new global rigidity theorem for such actions. During the conference (in fact, during discussions by the unruly audience of the talks!) the following theorem was deduced:

THEOREM 4.1 (Theorem 2 [32]) Let $\varphi: \Gamma \times X \rightarrow X$ be an Anosov $C^{r}$ action on a compact $n$-manifold $X$ without boundary, for $r=1, \infty$ or $\omega$. Suppose that $\Gamma$ satisfies Kazhdan's property $T$, and the action $\varphi$ preserves an absolutely continuous probability measure on $X$. Then there exists $\epsilon>0$ so that if $\varphi_{1}$ is $\epsilon-C^{1}$-close to $\varphi$, then $\varphi_{1}$ also preserves a $C^{r}$-volume form on $X$.

The point of this is that given an Anosov action of a higher rank lattice with a smooth invariant volume form, every $C^{1}$-close perturbation preserves an invariant volume form! Thus, one of the hypotheses of the Lewis-Zimmer theorem was eliminated for perturbations of the standard action. One can then deduce the smooth rigidity of the standard action of a finite-index subgroup $\Gamma \subset S L(3, \mathbf{Z})$ on $\mathbf{T}^{3}$, which had remained open till that point. The Lewis and Zimmer approach is detailed in the paper [35]. 
Cocycle super-rigidity can be combined with the study of abelian Cartan actions to obtain further classification of Anosov actions of higher rank lattices, an approach first described in the preprint [32] and refined in the author's paper [29]. First, we make a definition: A $C^{r}$-action $\varphi: \Gamma \times X \rightarrow X$ is said to be Cartan if there exists an abelian subgroup $\mathcal{A} \subset \Gamma$ generated (not necessarily freely!) by $\Delta=\left\{\gamma_{1}, \ldots, \gamma_{n}\right\} \subset \Gamma$, such that the restriction of $\varphi$ to $\mathcal{A}$ is an abelian Cartan action on $X$.

THEOREM 4.2 (Theorem 1 [29]) Let $\varphi: \Gamma \times \mathbf{T}^{n} \rightarrow \mathbf{T}^{n}$ be a Cartan $C^{r}$ action on the $n$-torus $\mathbf{T}^{n}$, for $r=1, \infty$ or $r=\omega$ in the real analytic case. Suppose that $\Gamma$ is a higher rank lattice and the subgroup $\mathcal{A} \subset \Gamma$ generated by $\Delta$ is a cocompact lattice in a maximal $\mathbf{R}$-split torus in $G$. If the action $\varphi$ preserves an absolutely continuous probability measure on $\mathbf{T}^{n}$, then $\varphi$ is $C^{r}$-conjugate to an affine action.

Combining Theorems 4.1 and 4.2 with the results about abelian Cartan actions of the last section we obtain:

COROLLARY 4.3 Let $\varphi: \Gamma \times \mathbf{T}^{n} \rightarrow \mathbf{T}^{n}$ be a Cartan $C^{r}$-action on the $n$ torus $\mathbf{T}^{n}$, for $r=1, \infty$ or $r=\omega$ in the real analytic case. Suppose that $\Gamma$ is a higher rank lattice and the subgroup $\mathcal{A} \subset \Gamma$ generated by the $\Delta$ is a cocompact lattice in a maximal $\mathbf{R}$-split torus in $G$. If the action $\varphi$ preserves an absolutelycontinuous probability measure on $\mathbf{T}^{n}$, then there exists $\epsilon>0$ so that if $\varphi_{1}$ is $\epsilon-C^{1}$-close to $\varphi$, then $\varphi_{1}$ is $C^{r}$-conjugate to the action $\varphi$.

As an example of this Corollary, let $\varphi: \Gamma \times \mathbf{T}^{n} \rightarrow \mathbf{T}^{n}$ be a standard action, and suppose that either:

1. $\Gamma \subset S L(n, \mathbf{Z})$ is a subgroup of finite index for $n \geq 3$; or

2. $\Gamma \subset S p(n, \mathbf{Z}) \subset S L(2 n, \mathbf{Z})$ is a subgroup of finite index of the group of integer symplectic matrices $S p(n, \mathbf{Z})$, for $n \geq 2$; or

3. $\Gamma$ is a subgroup of finite-index of a product $\Gamma_{0} \times \cdots \times \Gamma_{d} \subset S L(n, \mathbf{Z})$, where each factor group $\Gamma_{i}$ satisfies one of the two above cases, and $\Gamma$ contains a hyperbolic element.

Then $\varphi$ is $C^{r}$-rigid for $r=1, \infty$ and for $r=\omega$.

The key point to the proof of Theorem 4.2 is technical extension of a consequence of cocycle super-rigidity for Cartan actions (cf. Corollary 1.2 [77]):

PROPOSITION 4.4 Let $\varphi: \Gamma \times \mathbf{T}^{n} \rightarrow \mathbf{T}^{n}$ be a Cartan $C^{1}$-action on the $n$-torus $\mathbf{T}^{n}$ which preserves a smooth volume form. Suppose that $\Gamma$ is a higher rank lattice and the subgroup $\mathcal{A} \subset \Gamma$ generated by $\Delta$ is a cocompact lattice in a maximal $\mathbf{R}$-split torus in $G$. Then there exist

- continuous vector fields $\left\{\vec{v}_{1}, \ldots, \vec{v}_{n}\right\}$ on a finite covering of $\mathbf{T}^{n}$ which give a framing of the tangent bundle,

- a finite-index subgroup $\Gamma^{\prime} \subset \Gamma$, and

- a lift $\tilde{\varphi}$ of the restricted action $\varphi \mid \Gamma^{\prime}$ to this cover, 
so that with respect to this framing, the derivative cocycle $D \tilde{\varphi}: \Gamma^{\prime} \times \mathbf{T}^{n} \rightarrow$ $S L(n, \mathbf{R})$ is given by a homomorphism $\rho_{\varphi}: \Gamma^{\prime} \rightarrow S L(n, \mathbf{R})$.

From Proposition 4.4, we deduce that the restriction of the lifted action $\tilde{\varphi}$ to a finite-index subgroup of $\mathcal{A}$ has constant exponents at the periodic points, and therefore is smoothly equivalent to an affine action. The condition on the derivatives of the full lifted action of $\Gamma^{\prime}$ shows that this is also affine, from which the conclusions of Theorem 4.2 are deduced.

The hypotheses of Theorem 4.2 are clearly too strong, and more general results are certainly possible. For example, the techniques of proof all are true for actions on infra-nilmanifolds, so the result could have been stated in the greater generality where $\Gamma$ acts on a infra-nilmaniofld $X$. As a second example, the hypothesis on the invariant measure can be replaced by an assumption on the induced homotopy action:

THEOREM 4.5 ([29]) Let $\varphi: \Gamma \times X \rightarrow X$ be an Anosov $C^{r}$-action on a compact nilmanifold $X$, for $r=1, \infty, \omega$. Suppose that $\Gamma$ is a higher rank lattice and the subgroup $\mathcal{A} \subset \Gamma$ generated by $\Delta$ is a cocompact lattice in a maximal $\mathbf{R}$-split torus in $G$. Suppose that the induced algebraic action of $\Gamma$ on the nilmanifold $\hat{\pi}_{1}(\tilde{X}) / \pi_{1}(\tilde{X})$ obtained from the Mal'cev completion is Cartan. Then $\varphi$ is $C^{r}$-conjugate to an affine action.

Further applications of this method are certain to be developed, so the reader must regard this report as the situation as of summer 1992.

\section{$5 \quad$ Standard examples}

The purpose of this section is to discuss some of the examples of algebraic lattice group actions on $\mathbf{T}^{n}$ which are rigid by the theorems of the previous sections. The list is taken from section 7 of [28], and should give the reader an idea of the available constructions. The proofs of the propositions and lemmas cited below can be found there. Also, we do not go into examples of lattice actions on nilmanifolds; this is discussed in [29]. Here is an overview of the examples:

- finite index subgroups of the integer matrices acting on the torus;

- subgroups of the integer symplectic matrices.

- lattices obtained from Weyl's technique of restriction of scalars. (The issue with these examples is to obtain the Anosov condition.)

- combinations of these three types of examples via the constructions of geometric sums, products, diagonal actions and "arithmetic products".

We begin by recalling some of the standard facts regarding lattices. The fundamental result on the existence of lattices is due to Borel and HarishChandra ([6]; cf. also Chapter XIV, [56].)

THEOREM 5.1 (Borel-Harish-Chandra) Let $G \subset S L(N, \mathbf{C})$ be a semisimple algebraic group defined over $\mathbf{Q}$. Then the group of integer points $G_{\mathbf{Z}}$ is a lattice in the group of real points $G_{\mathbf{R}}$. 
The group $\Gamma=G_{\mathbf{Z}}$ preserves the integer lattice in $\mathbf{R}^{N}$, so descends to a standard action on $\mathbf{T}^{N}$.

The Margulis Vanishing Theorem 2.2 discussed in section 2 implies that $\mathrm{SVC}(\mathrm{N})$ holds for all $N \geq 1$ for every subgroup $\Gamma \subset G_{\mathbf{Z}}$ of finite index, where $G$ is as in Theorem 5.1, $\left(G_{\mathbf{R}}\right)_{0}$ has no compact factors, and $G_{\mathbf{R}}$ has $\mathbf{R}$-split rank at least 2 .

EXAMPLE $1(\mathbf{S L}(\mathbf{n}, \mathbf{Z}))$ Let $(\mathbf{Q} / \mathbf{Z})^{n}$ denote the rational torus in $\mathbf{T}^{n}$. The following is an easy exercise.

LEMMA 5.2 Let $\Gamma \subset S L(n, \mathbf{Z})$ contain a hyperbolic element for the standard action of $\Gamma$ on $\mathbf{T}^{n}$. Then the periodic points of the standard action of $\Gamma$ on $\mathbf{T}^{n}$ are $\Lambda=(\mathbf{Q} / \mathbf{Z})^{n}$, and hence are dense.

For a subgroup $\Gamma \subset S L(n, \mathbf{Z})$ of finite index for $n \geq 3$, the Margulis Vanishing Theorem 2.2 implies that SVC(N) holds for all $N \geq 1$.

The Prasad \& Raghunathan result (Theorem 3.13 above, or Theorem 2.8 and Corollary 2.9 [55]) yields the following:

LEMMA 5.3 Let $\Gamma \subset S L(n, \mathbf{Z})$ be a subgroup of finite index. Then there a subset of commuting hyperbolic elements, $\Delta \subset \Gamma$, so that each $\gamma_{i} \in \Delta$ has a 1dimensional contracting eigenspace $E_{i} \subset \mathbf{R}^{n}$ with internal direct sum $E_{1} \oplus \cdots \oplus$ $E_{n} \cong \mathbf{R}^{n}$. Consequently, the standard action of $\Gamma$ on $\mathbf{T}^{n}$ is maximal Cartan.

It is a standard question to produce explicit matrices of the form promised by the Prasad \& Raghunathan theorem. This is accomplished using methods of number theory. Our thanks to Leon McCullough for the following example:

LEMMA 5.4 The following matrices in $S L(3, \mathbf{Z})$

$$
A_{1}=\left[\begin{array}{l}
-2,1,0 \\
-1,0,1 \\
-2,1,1
\end{array}\right] \quad A_{2}=\left[\begin{array}{l}
1,-2,1 \\
0,-2,1 \\
1,-1,0
\end{array}\right] \quad A_{3}=\left[\begin{array}{l}
0,1,-1 \\
1,1,-2 \\
1,0,-2
\end{array}\right]
$$

are hyperbolic, satisfy the identities:

$$
\begin{aligned}
& A_{1} A_{2}=A_{2} A_{1}=A_{1}+A_{2} \\
& A_{1} A_{3}=A_{3} A_{1}=A_{2}+A_{3} \\
& A_{2} A_{3}=A_{3} A_{2}=A_{1}+A_{2}
\end{aligned}
$$

and are roots of the characteristic polynomial $x^{3}+x^{2}-2 x-1=0$.

COROLLARY 5.5 Let $\Gamma \subset S L(n, \mathbf{Z})$ be a subgroup of finite-index. Then the standard action of $\Gamma$ on $\mathbf{T}^{n}$ is $C^{r}$-rigid for $r=1, \infty, \omega$.

EXAMPLE $2(\mathbf{S p}(\mathbf{n}, \mathbf{Z}))$ The previous example for $S L(n, \mathbf{Z})$ corresponds to the "A" series of simple Lie groups. There are corresponding Anosov actions for the symplectic groups, or the "C" series. The rigidity of the standard action of one Anosov element in $S p(2, \mathbf{Z})$ was studied in [14].

The strength of the Prasad-Raghunathan theorem is illustrated by its application in the symplectic case: 
LEMMA 5.6 Let $n \geq 1$ and let $\Gamma \subset S p(n, \mathbf{Z})$ be a subgroup of finite index. Then there exists commuting matrices $\delta_{1}, \ldots, \delta_{n} \in \Gamma$ such that the set $\Delta=$ $\left\{\delta_{1}, \delta_{1}^{-1}, \ldots, \delta_{n}, \delta_{n}^{-1}\right\}$ generates an abelian subgroup $\mathcal{A}$ whose standard action on $\mathbf{T}^{2 n}$ is trellised.

COROLLARY $\mathbf{5 . 7}$ Let $\Gamma \subset S p(n, \mathbf{Z})$ be a subgroup of finite-index. Then the standard action of $\Gamma$ on $\mathbf{T}^{2 n}$ is $C^{r}$-rigid for $r=1, \infty, \omega$.

EXAMPLE $3(\mathbf{S L}(\mathbf{n}, \mathcal{O}(\mathbf{k})))$ Let $\mathbf{k} \subset \mathbf{R}$ be an algebraic number field of degree $d$ over $\mathbf{Q}$, let $\mathcal{O}(\mathbf{k})$ be the ring of integers for the field and let $S L(n, \mathcal{O}(\mathbf{k}))$ be the subgroup of $S L(n, \mathbf{k})$ with entries from $\mathcal{O}(\mathbf{k})$. The "Restriction of Scalars" technique of $\mathrm{A}$. Weil yields a wide range of lattice actions.

PROPOSITION 5.8 For $n \geq 2$ and $\Gamma \subset S L(n, \mathcal{O}(\mathbf{k}))$ a subgroup of finite index,

1. there exists an analytic "standard" action of $\Gamma$ on $\mathbf{T}^{d n}$, and

2. if the group $G_{\mathbf{R}}=R_{\mathbf{k} / \mathbf{Q}}(S L(n, \mathbf{R}))_{\mathbf{R}}$ of real points (for the group $G$ obtained by the restriction of scalars) has no compact factors, then the standard action of $\Gamma$ is Anosov.

Proof. We recall the proof of this, as the technique is not well-known (cf. pages 115-116, [71].) Let $\left\{\sigma_{1}, \ldots, \sigma_{d}\right\}$ be distinct field embeddings of $\mathbf{k}$ into $\mathbf{R}$ with $\sigma_{1}$ the identity inclusion. Each embedding $\sigma_{i}$ defines a map $\sigma_{i}^{n}: \mathbf{k}^{n} \rightarrow \mathbf{R}^{n}$, and so we get a $\mathbf{Q}$-linear map

$$
\begin{aligned}
\sigma^{n}: \mathbf{k}^{n} & \longrightarrow \mathbf{R}^{d n} \\
\sigma^{n}(w) & =\left(\sigma_{1}(w), \ldots, \sigma_{d}(w)\right)
\end{aligned}
$$

whose extension to $\mathbf{R}$ over $\mathbf{Q}$ is an isomorphism. This induces an isomorphism of $S L(n, \mathbf{k})$ with an algebraic subgroup $G \subset S L(d n, \mathbf{R})$ which is defined over $\mathbf{Q}$. The image of the group $S L(n, \mathcal{O}(\mathbf{k}))$ is then seen to equal the integral points $G_{\mathbf{Z}}$ of $G$. We define the standard action of $S L(n, \mathcal{O}(\mathbf{k}))$ on $\mathbf{T}^{d n}$ via this embedding.

The group $G$ defined over $\mathbf{k}$ is equal to the product of the embeddings $G^{\sigma_{i}}=\sigma_{i}(S L(n, \mathbf{k}))$, and the set of real points has a similar product structure

$$
G_{\mathbf{R}} \cong \prod_{i=1}^{d}\left(G^{\sigma_{i}}\right)_{\mathbf{R}}
$$

The image of $\sigma^{n}(\Gamma) \subset G_{\mathbf{R}}$ is a lattice by Weil's theory of restriction of scalars, so that if no factor $\left(G^{\sigma_{i}}\right)_{\mathbf{R}}$ is compact, then we can find a Cartan subgroup for $G_{\mathbf{R}}$ containing a hyperbolic element for the standard action. Then by Theorem 3.13 of Prasad and Raghunathan, the image of $\Gamma$ will contain a hyperbolic element.

The usual application of Weil's theory of restriction of scalars is to produce cocompact lattices in an arithmetic Lie group (cf. Example 6.1.5, [71], or page $216,[56])$. In these constructions, the field extension has degree 2 , with $\left(G^{\sigma_{1}}\right)_{\mathbf{R}}$ isomorphic to $(S L(n, \mathbf{k}))_{\mathbf{R}}$ and $\left(G^{\sigma_{2}}\right)_{\mathbf{R}}$ isomorphic to a compact Lie group. These examples do not give Anosov standard actions. 
COROLLARY 5.9 Let $n \geq 3$ and let $k$ be an algebraic number field of degree $d$ over $\mathbf{Q}$, such that the group $R_{\mathbf{k} / \mathbf{Q}}(S L(n, \mathbf{k}))_{\mathbf{R}}$ has no compact factor. For any subgroup $\Gamma \subset S L(n, \mathcal{O}(\mathbf{k}))$ of finite index, the standard action of $\Gamma$ on $\mathbf{T}^{d n}$ is $C^{r}$-rigid for $r=1, \infty, \omega$.

EXAMPLE 4 (Geometric Sums and Products) Let $\left\{\varphi_{i}: \Gamma_{i} \times X_{i} \rightarrow X_{i}\right.$ $1 \leq i \leq d\}$ be given $C^{r}$-actions. Then the direct product action of $\Gamma=\Gamma_{1} \times$ $\cdots \times \Gamma_{d}$ on $X=X_{1} \times \cdots \times X_{d}$ is obtained by letting the subgroup $\Gamma_{i}$ act on the factor $X_{i}$ via $\varphi_{i}$ and via the identity on $X_{j}$ for $j \neq i$, and then extending to all of $\Gamma$ via products. The following is easy to check:

LEMMA 5.10 Suppose that each action $\varphi_{i}$ for $1 \leq i \leq d$ is Anosov (respectively trellised, Cartan). Then the direct product action $\varphi: \Gamma \times X \rightarrow X$ is Anosov (respectively trellised, Cartan).

COROLLARY 5.11 For $1 \leq i \leq d$, let $\Gamma_{i} \subset S L\left(n_{i}, \mathbf{Z}\right)$ be isomorphic to a higher rank lattice, and so that the standard action $\varphi_{i}: \Gamma_{i} \times \mathbf{T}^{n_{i}} \rightarrow \mathbf{T}^{n_{i}}$ is Cartan with linear trellising. Then for any finite-index subgroup $\Gamma \subset \Gamma_{1} \times \cdots \times \Gamma_{d}$, the restricted product action $\varphi=\varphi_{1} \times \cdots \times \varphi_{d}$ of $\Gamma$ on $\mathbf{T}^{n}=\mathbf{T}^{n_{1}} \times \cdots \times \mathbf{T}^{n_{d}}$ is $C^{r}$-rigid for $r=1, \infty, \omega$.

Suppose that each space $X_{i}=\mathbf{T}^{n_{i}}$ for integers $n_{i}>2$, and $\Gamma_{i} \subset S L\left(n_{i}, \mathbf{Z}\right)$. The geometric tensor product of the standard actions $\left\{\varphi_{i}: \Gamma_{i} \times \mathbf{T}^{n_{i}} \rightarrow \mathbf{T}^{n_{i}} \mid 1 \leq\right.$ $i \leq d\}$ is obtained by taking the induced action of the lattices $\Gamma_{i}$ on the tensor product $\mathbf{R}^{n_{1}} \otimes \cdots \otimes \mathbf{R}^{n_{d}}$, and observing that this preserves the tensor product lattice $\mathbf{Z}^{n_{1}} \otimes \cdots \otimes \mathbf{Z}^{n_{d}}$. We obtain the tensor product action $\varphi$ of $\Gamma_{1} \times \cdots \times \Gamma_{d}$ on $\mathbf{T}^{N}$ where $N=n_{1} \cdots n_{d}$. Then for any finite-index subgroup $\Gamma \subset \Gamma_{1} \times \cdots \times \Gamma_{d}$ :

LEMMA 5.12 Suppose that each action $\varphi_{i}$ for $1 \leq i \leq d$ is Anosov (respectively trellised). Then the restricted tensor product action $\varphi: \Gamma \times \mathbf{T}^{N} \rightarrow \mathbf{T}^{N}$ is Anosov (respectively trellised).

Remark: Note that a tensor product action will never be a Cartan action, as it is impossible to have a basis of maximally contracting eigenspaces.

COROLLARY 5.13 For $1 \leq i \leq d$, let $\Gamma_{i} \subset S L\left(n_{i}, \mathbf{Z}\right)$ be isomorphic to a higher rank lattice, and so that the standard action $\varphi_{i}: \Gamma_{i} \times \mathbf{T}^{n_{i}} \rightarrow \mathbf{T}^{n_{i}}$ is linearly trellised. Then the restricted tensor product action $\varphi=\varphi_{1} \otimes \cdots \otimes \varphi_{d}$ of $\Gamma$ on $\mathbf{T}^{N}$, for $N=n_{1} \cdots n_{d}$, is $C^{0, r}$-deformation rigid for $r=1, \infty, \omega$.

EXAMPLE 5 (Diagonal Actions) The $d$-fold diagonal action of an action $\varphi: \Gamma \times X \rightarrow X$ is obtained by restricting the product action of $d$-copies of $\varphi$ to the $d$-fold diagonal. There is a slightly more general construction available. Let actions $\left\{\varphi_{i}: \Gamma \times X_{i} \rightarrow X_{i} \mid 1 \leq i \leq d\right\}$ be given, then we obtain an action of $\Gamma$ on $X=X_{1} \times \cdots \times X_{d}$ by setting

$$
\varphi(\gamma)\left(x_{1}, \ldots, x_{d}\right)=\left(\varphi_{1}(\gamma)\left(x_{1}\right), \ldots, \varphi_{d}(\gamma)\left(x_{d}\right)\right)
$$


LEMMA 5.14 Let $\varphi$ be the generalized diagonal action obtained from the Anosov actions $\left\{\varphi_{i} \mid 1 \leq i \leq d\right\}$. If there exists $\gamma \in \Gamma$ such that $\gamma$ is $\varphi_{i}$-hyperbolic for all $1 \leq i \leq d$, then $\varphi$ is an Anosov action.

COROLLARY 5.15 Let $\left\{\varphi_{i}: \Gamma \times X_{i} \rightarrow X_{i} \mid 1 \leq i \leq d\right\}$ be Anosov actions with dense periodic orbits, with a common hyperbolic element $\gamma$. If $\Gamma$ satisfies the cohomology condition $S V C\left(n^{2}\right)$ for $n=n_{1}+\cdots+n_{d}$, then $\varphi$ is $C^{k}$-topologically deformation rigid.

A diagonal action with $\phi_{i}=\phi$ the same for all $i$ can not be Cartan for $d \geq 2$, as the dimensions of the eigenspaces for the hyperbolic elements are always at least $d$; hence the strongest stable direction is always of dimension at least $d$.

Diagonal actions provide a large collection of examples where topological deformation rigidity is the best result known. It seems difficult, at the present state of research, to decide whether these actions are differentiably rigid. A natural test case is to show they are $C^{1}$-deformation rigid; for example, by studying the properties of cocycles over product actions.

EXAMPLE 6 (Arithmetic Products) Let $\left\{\varphi_{i}: \Gamma_{i} \times \mathbf{T}^{n_{i}} \rightarrow \mathbf{T}^{n_{i}} \mid 1 \leq i \leq\right.$ $d\}$ be Anosov standard actions of arithmetic subgroups $\Gamma_{i}=\left(G_{i}\right)_{\mathbf{z}}$, where $G_{i} \subset S L\left(n_{i}, \mathbf{R}\right)$ is a connected semi-simple algebraic group defined over $\mathbf{Q}$, with real-rank at least 2 . There is an alternate construction of a standard action of a group $\Gamma$ on a torus constructed from this data, that we call the arithmetic product.

The product group $G=G_{1} \times \cdots \times G_{d} \subset S L(n, \mathbf{R})$ is defined over $\mathbf{Q}$, for $n=n_{1}+\cdots+n_{d}$. The group of real points $G_{\mathbf{R}}$ admits an arithmetic irreducible lattice subgroup $\Gamma \subset G_{\mathbf{R}}$. That is, for some $N \geq n$ there exists a group $\tilde{G} \subset S L(N, \mathbf{R})$ containing a lattice $\tilde{\Gamma}=\tilde{G}_{\mathbf{Z}}$, and there is a natural homomorphism $\pi: \tilde{G} \rightarrow G$ whose restriction to $\tilde{\Gamma}$ is an isomorphism.

The arithmetic product of the actions $\left\{\varphi_{i}\right\}$ is the action of $\Gamma$ on $\mathbf{T}^{N}$ via the inverse map

$$
\left(\left.\pi\right|_{\tilde{\Gamma}}\right)^{-1}: \Gamma \longrightarrow S L(N, \mathbf{Z})
$$

This construction is similar to Example 3. To determine whether such an action is Anosov or Cartan, we first must determine whether $\tilde{G}$ contains a nontrivial compact factor. This entails a more extensive discussion of cases, which we omit.

\section{Non-standard examples}

An area of mathematics needs a wealth of examples to flourish; after seeing some of the available algebraic constructions of standard examples in the last section, we conclude this survey with a collection of non-standard examples. Again, this is not an exhaustive list, but should suggest to the reader the possibilities, and hopefully motivate the construction of further examples. Examples are given of: 
- a deformation of the standard action of $S L(2, \mathbf{Z})$

- non-Anosov but almost everywhere hyperbolic lattice actions (after Katok \& Lewis)

- affine actions with no fixed-points

- normally hyperbolic actions

and conclude with a "wish list" of examples we would like to see.

EXAMPLE 7 (A deformation of the standard action of SL(2,Z)) We construct a 1-parameter family of real analytic actions of $S L(2, \mathbf{Z})$ on $\mathbf{T}^{2}$ which is not topologically deformation rigid. Thus, the Anosov hypotheses is not sufficient for the topological rigidity of a group action with more than one generator and additional hypotheses are necessary to obtain rigidity.

THEOREM 6.1 (Theorem 7.22 [28]) There exists an analytic family $\left\{\varphi_{t} \mid\right.$ $0 \leq t \leq 1\}$ of volume-preserving real analytic actions of $S L(2, \mathbf{Z})$ on $\mathbf{T}^{2}$, with $\varphi_{0}=\varphi$ the standard action, such that $\varphi_{t}$ is not topologically conjugate to $\varphi$ for all $0<t \leq 1$.

Proof. First recall:

LEMMA 6.2 1. The pair of matrices $A=\left[\begin{array}{cc}0 & -1 \\ 1 & 0\end{array}\right]$ and $B=\left[\begin{array}{cc}1 & -1 \\ 1 & 0\end{array}\right]$ generate $S L(2, \mathbf{Z})$.

2. $A$ has order $4, B$ has order 6 , and $A^{2}=B^{3}=-I$.

3. $S L(2, \mathbf{Z})$ is isomorphic to the amalgamated product $(\mathbf{Z} / 4 \mathbf{Z}) \underset{\mathbf{Z} / 2 \mathbf{Z}}{\times}(\mathbf{Z} / 6 \mathbf{Z})$ generated by $\{A, B\}$.

Our construction is based on deforming the action of one of these generators using the flow of a vector field. The deformation is required to preserve the relations satisfied by $A$ and $B$. The proof that the new actions are not standard then follows from analyzing the invariant manifolds for a particular element. Folkert Tangerman has constructed similar deformations from a geometric viewpoint, and described the construction as "opening up an invariant circle". Thus, this is a kind of "lattice-action earthquake", in reference to Thurston's method of deforming hyperbolic structures.

Let $\vec{Z}_{1}=x \frac{\partial}{\partial y}-y \frac{\partial}{\partial x}$ be the rotational vector field about the origin. Then for any smooth function $\psi(s)$, the vector field $\vec{Z}_{\psi}=\psi\left(x^{2}+y^{2}\right) \cdot \vec{Z}_{1}$ is divergence free.

We first form a non-trivial family of $C^{\infty}$-deformations, then indicate the modifications necessary for the real analytic case. Choose a smooth function $\psi$ such that $\psi(0)=1, \psi(s) \geq 0$ for all $s$, and $\psi(s)=0$ for $s \geq 10^{-4}$. Form the translate of the vector field $\vec{Z}_{\psi}$, centered at the point $[1 / 2,0] \in \mathbf{R}^{2}$ :

$$
Z_{+}=D T_{[1 / 2,0]}\left(Z_{\psi}\right)
$$


Introduce the companion vector field $Z_{-}=D\left(A^{2}\right)\left(Z_{+}\right)=D(-I)\left(Z_{+}\right)$, and form the sum $Z=Z_{+}+Z_{-}$. Note that $D\left(A^{2}\right)(Z)=Z$.

We want the vector field $Z$ to be invariant under the translation action of the lattice $\mathbf{Z}^{2}$, so we form the infinite sum

$$
\tilde{Z}=\sum_{[m, n] \in \mathbf{Z}} D T_{[m, n]}(Z)
$$

which is well-defined since the supports of the translates are disjoint.

Let $F(t): \mathbf{R}^{2} \rightarrow \mathbf{R}^{2}$ be the flow of the vector field $\tilde{Z}$, and observe that

$$
\begin{aligned}
F(t) \circ A^{2} & =A^{2} \circ F(t) \\
T_{[m, n]} \circ F(t) & =F(t) \circ T_{[m, n]} .
\end{aligned}
$$

From equation (8) the maps $F(t)$ descend to a family of diffeomorphisms of $\mathbf{T}^{2}$ denoted by $\tilde{F}(t)$. Moreover, from the identity (7) we have that

$$
\left\{\tilde{F}^{-1}(t) \circ \varphi(A) \circ \tilde{F}(t)\right\}^{2}=-I
$$

which by Lemma 6.2 implies there is a well-defined $C^{\infty}$-deformation of the standard action $\varphi$ of $S L(2, \mathbf{Z})$, by declaring that

$$
\begin{aligned}
\varphi_{t}(A) & =\tilde{F}(t)^{-1} \circ \varphi(A) \circ \tilde{F}(t) \\
\varphi_{t}(B) & =\varphi(B)
\end{aligned}
$$

On can then check that this family of deformations is non-trivial:

LEMMA 6.3 ([28]) If there exists a homeomorphism $H: \mathbf{T}^{2} \rightarrow \mathbf{T}^{2}$ conjugating $\varphi_{t}$ to $\varphi_{0}$, then $t=0$.

Analytic deformations are easily obtained by using the cut-off function $\psi(s)=\exp \left\{-(100 s)^{2}\right\}$. The support of the exponential function is no longer compact, but the sum (6) will still yield an analytic vector field, for the index set grows linearly with the weight $|n|+|m|$, and the function $\exp \left\{-10000\left(n^{2}+m^{2}\right)\right\}$ decays super-exponentially fast in this weight.

EXAMPLE 8 (Non-affine ergodic lattice actions) Katok and Lewis describe in section 4 of [33] a family of examples of actions on compact manifolds by a higher rank lattices, which are smooth, volume-preserving and ergodic, but not affine. The constructions are "elementary", in that the authors take a standard action and "blow-up" some of the periodic orbits. To produce an invariant volume form for the blow-up requires a change in the smooth structure on the manifold in a neighborhood of the divisor along which the blow-up occurs. Thus, the construction is actually quite sophisticated in its overall approach. These examples are notable for while they are derived from linear actions, the end result is not affine. Moreover, there is a great latitude available in the construction, which suggests that these type of ideas will be very rewarding of further development. Katok and Lewis conjecture that this sort of example 
is the "typical case" for ergodic actions of higher rank lattices, Conjecture 1.1 [33]. This would fit perfectly with M. Gromov's program to study the invariant geometric structures of large group actions [17].

Let us describe intuitively the idea of Katok and Lewis' construction. The action of $G L(n, \mathbf{R})$ on $\mathbf{R}^{n}$ fixes the origin 0 and is $C^{1}$ near 0 , hence induces an action on the blow-up of $\mathbf{R}^{n}$ at 0 . (For details on the blow-up construction, see for example the book by Griffiths and Harris, [16].) Restricting this construction to a lattice subgroup $\Gamma \subset S L(n, \mathbf{Z}) \subset G L(n, \mathbf{R})$, we see that there is an induced action of $\Gamma$ on the blow-up of this fixed-point.

Given a higher-rank lattice $\Gamma \subset S L(n, \mathbf{Z})$, there is a finite set of fixed-points for a standard Anosov action on $\mathbf{T}^{n}$. Choose a subset $\left\{x_{i}, \ldots, x_{d}\right\}$ of the fixedpoints, then about each $x_{i}$ we can perform (locally) the blow-up construction. Let $M$ denote the resulting real-analytic manifold obtained from inductively blowing up this set of points, $\mathbf{T}^{n}$, with $\left\{Z_{1}, \ldots, Z_{d}\right\}$ the codimension-one analytic subvarieties corresponding to the blow-up of the points. Then we obtain a real analytic action of $\Gamma$ on $M$ for which the submanifolds $Z_{i}$ are invariant. Moreover, the action on the complement

$$
M-\left\{Z_{1} \cup \cdots \cup Z_{d}\right\} \cong \mathbf{T}^{n}-\left\{x_{1}, \ldots, x_{d}\right\}
$$

is equivalent to the linear action. It follows that the action of $\Gamma$ on $M$ is ergodic and "uniformly Anosov" on a dense open subset.

The volume form on $\mathbf{T}^{n}$ restricts to one on $\mathbf{T}^{n}-\left\{x_{1}, \ldots, x_{d}\right\}$ and hence there is a smooth invariant density on $M$. Unfortunately, this form is zero on the divisors $\left\{Z_{1} \cup \cdots \cup Z_{d}\right\}$. Katok and Lewis then show that by a change of coordinates in the normal bundle to these divisors, the singular volume form becomes non-singular and invariant for the resulting action of $\Gamma$. This is a very delicate construction, and the reader is referred to page 18 of [33] for details.

This construction of Katok and Lewis is similar to one used by O. H. Rasmussen to construct new examples of group actions on the two-sphere, whose secondary classes are independently variable [57]. Away from the theme of this paper, it is interesting to speculate whether the above examples have non-trivial characteristic invariants associated with them, in the spirit of $[21,23]$ ?

The projectivizing of the linear actions of lattices was also the key technique for J. Heitsch's construction of families of group actions whose secondary classes varied continuously and independently [22]. The Heitsch construction yields continuous families of actions, and is easily adapted to the situation of Katok and Lewis. Thus, one can obtain continuous families of lattice actions which are presumably not smoothly equivalent, by reason of characteristic class techniques. They are conjecturally not topologically equivalent either.

EXAMPLE 9 (Affine actions without fixed-points) The standard actions of subgroups of $S L(n, \mathbf{Z})$ on $\mathbf{T}^{n}$ all fix the coset of the origin. The problem of classifying the Anosov actions of higher rank lattices raises the question whether a lattice action that preserves an affine structure must have a fixedpoint? A periodic point? The existence of a fixed-point is a problem concerning 
the torsion elements in a certain cohomology group associated to the action. The following is an example of actions for which this group is non-trivial.

THEOREM 6.4 (Theorem 1 [27]) For each $n \geq 2$ and $p>1$, there exists a lattice subgroup $\Gamma(n, p) \subset S L(n, \mathbf{Z})$ and an affine action $\tilde{\varphi}$ with linear part given by the standard action of $\Gamma(n, p)$ on $\mathbf{T}^{n}$, such that:

1. $S L(n, \mathbf{Z})_{p^{2}} \subset \Gamma(n, p) \subset S L(n, \mathbf{Z})_{p}$, where $S L(n, \mathbf{Z})_{p}$ denotes the congruence p-subgroup;

2. the restricted action of $\tilde{\varphi}$ to $S L(n, \mathbf{Z})_{p^{2}}$ is the standard linear action;

3. the affine action $\tilde{\varphi}$ of $\Gamma(n, p)$ has dense periodic orbits, but has no fixedpoints.

Our construction produces groups $\Gamma(n, p) \subset S L(n, \mathbf{Z})$ with non-trivial torsion classes in $H^{1}\left(\Gamma(n, p) ; \mathbf{T}_{\varphi_{0}}^{n}\right)$, where $\varphi_{0}$ is the standard linear action of $\Gamma(n, p)$ on $\mathbf{T}^{n}$. These classes then correspond to affine actions of $\Gamma(n, p)$ on $\mathbf{T}^{n}$ with periodic orbits, but no fixed-points.

Let $\mathbf{Z} / p \mathbf{Z}$ denote the cyclic group of order p. For each $n>1$, there is a natural "mod-p" quotient map $\Pi_{p}: S L(n, \mathbf{Z}) \rightarrow S L(n, \mathbf{Z} / p \mathbf{Z})$, whose kernel is the congruence-p subgroup denoted by $S L(n, \mathbf{Z})_{p}$. Given a subgroup $\Gamma \subset$ $S L(n, \mathbf{Z})$, define $\Gamma_{q}=\Gamma \cap S L(n, \mathbf{Z})_{p}$. Observe then that $\Gamma_{q}$ is normal with finite index in $\Gamma$.

Recall that $\varphi_{0}: \Gamma \times \mathbf{T}^{n} \rightarrow \mathbf{T}^{n}$ is the standard action. For a subgroup $\Gamma \subset S L(n, \mathbf{Z})$, define the subgroup of $\Gamma$-invariants in $\mathbf{T}^{n}$,

$$
\mathcal{I}(\Gamma)=\left(\mathbf{T}^{n}\right)^{\Gamma}=\left\{x \in \mathbf{T}^{n} \mid x=\varphi_{0}(\gamma)(x) \text { for all } \gamma \in \Gamma\right\}
$$

LEMMA 6.5 There is a natural map $\operatorname{Hom}(\Gamma, \mathcal{I}(\Gamma)) \stackrel{i_{*}}{\longrightarrow} H^{1}\left(\Gamma, \mathbf{T}_{\varphi_{0}}^{n}\right)$.

Proof. $\varphi_{0}$ restricts to the trivial action on $\mathcal{I}(\Gamma)$, so the inclusion $i: \mathcal{I}(\Gamma) \subset \mathbf{T}^{n}$ induces a well-defined map $\operatorname{Hom}(\Gamma, \mathcal{I}(\Gamma)) \cong H^{1}\left(\Gamma ; \mathcal{I}(\Gamma)_{\varphi}\right) \stackrel{i_{*}}{\longrightarrow} H^{1}\left(\Gamma, \mathbf{T}_{\varphi_{0}}^{n}\right)$.

Let $\frac{1}{p} \mathbf{Z}$ denote the additive group of fractions with denominator $\frac{1}{p}$, and let $\mathbf{T}_{p}^{n}=\left(\frac{1}{p} \mathbf{Z}\right)^{n} \bmod \mathbf{Z}^{n}$ be the " $1 /$ p-points" for the n-torus.

LEMMA 6.6 For each $p \geq 1, \mathcal{I}\left(S L\left(n, \mathbf{Z}_{p}\right)\right)=\mathbf{T}_{p}^{n}$

The proof of Theorem 6.4 follows by exhibiting a lattice subgroup $\Gamma(n, p)$ such that $S L(n, \mathbf{Z})_{p^{2}} \subset \Gamma(n, p) \subset S L(n, \mathbf{Z})_{p}$ and

$$
i_{*}: \operatorname{Hom}\left(\Gamma(\mathrm{n}, \mathrm{p}), \mathbf{T}_{\mathrm{p}}^{\mathrm{n}}\right) \longrightarrow H^{1}\left(\Gamma(n, p), \mathbf{T}_{\varphi}^{n}\right)
$$

is not trivial. Let $U_{a}$ denote the $n \times n$ matrix with all entries zero, except for the top right entry which equals $a$, and $I d$ be the $n \times n$ identity matrix. Then $A=I d+U_{p} \in S L(n, \mathbf{Z})_{p}$ is an upper triangular matrix. Note that $A^{p}=I d+U_{p^{2}}$, so that $A$ generates a cyclic subgroup of order $p$ in the quotient group $S L(n, \mathbf{Z})_{p} / S L(n, \mathbf{Z})_{p^{2}}$. Define $\Gamma(n, p)$ to be the group generated by $A$ and the subgroup $S L(n, \mathbf{Z})_{p^{2}}$. Let $\Pi: \Gamma(n, p) \rightarrow \mathbf{Z} / p \mathbf{Z}$ be the quotient map 
onto the cyclic group of order $p$, where the kernel of $\Pi$ is $S L(n, \mathbf{Z})_{p^{2}}$, and $\Pi(A)=1 \in \mathbf{Z} / p \mathbf{Z}$.

For $1 \leq i \leq n$, define homomorphisms $\tau_{i}: \Gamma(n, p) \rightarrow \mathbf{T}_{p}^{n}$ by

$$
\tau_{i}(\gamma)=\frac{\Pi(\gamma)}{p} \cdot \vec{e}_{i} \bmod \mathbf{Z}^{n}
$$

where $\vec{e}_{i}=(0, \ldots, 1, \ldots, 0)$ is the $i^{\text {th }}$ basis vector of $\mathbf{Z}^{n}$.

LEMMA 6.7 The elements $\left\{i_{*}\left[\tau_{1}\right], \ldots, i_{*}\left[\tau_{n-1}\right]\right\} \in H^{1}\left(\Gamma(n, p), \mathbf{T}_{\varphi_{0}}^{n}\right)$ are linearly independent over $\mathbf{Z} / p \mathbf{Z}$.

COROLLARY 6.8 There is an inclusion $(\mathbf{Z} / p \mathbf{Z})^{n-1} \subset H^{1}\left(\Gamma(n, p) ; \mathbf{T}_{\varphi_{0}}^{n}\right)$.

The points in the rational torus $\mathbf{Q}^{n} / \mathbf{Z}^{n}$ are all periodic for the standard action, and also for the translation action by a rational number. It follows that $\mathbf{Q}^{n} / \mathbf{Z}^{n}$ is contained in the set of periodic orbits for each affine action $\varphi_{\tau}$ associated to a class $[\tau]=a_{1} \cdot\left[\tau_{1}\right]+\cdots+a_{n-1} \cdot\left[\tau_{n-1}\right] \in H^{1}\left(\Gamma(n, p), \mathbf{T}_{\varphi}^{n}\right)$, hence $\Lambda\left(\varphi_{\tau}\right)$ is dense. When $[\tau] \neq 0$, there are no fixed-points for the affine action $\varphi_{\tau}$. Theorem 6.4 now follows.

A more general formulation of this construction of affine actions is given in section 4 of [27] where the cohomology methods above are replaced with their dynamical counter-parts.

EXAMPLE 10 (Normally hyperbolic actions) There are a plethora of constructions of lattice actions which are algebraic but not Anosov. For example, the typical examples studied by R. Zimmer [73, 75, 68] are of this type: take a cocompact lattice $\Lambda \subset G$ in a semi-simple Lie group $G$ and form the compact quotient $M=G / \Lambda$. Then every homomorphism $\rho: \Gamma \rightarrow G$ induces a left action $\hat{\rho}$ of $\Gamma$ on $M$. The tangent bundle $T M$ admits a framing by the quotient of a right-invariant framing of $T G$, and the derivative action of an element $g \in G$ is described in terms of the adjoint representation $\operatorname{Ad}(g) \in$ $G L\left(T_{e} G\right)$. For a semi-simple connected Lie group $G$, the matrix $A d(g)$ always centralizes at least one direction in $T_{e} G$, so that the action $\hat{\gamma}$ is never Anosov for $\gamma \in \Gamma$. On the other hand, these actions are often normally hyperbolic with respect to a foliation of $M$, in the sense of Chapter 7 of [45].

There is a tremendous interest in extending the methods of Anosov actions described in this survey, to the normally-hyperbolic examples just described. This would give a dynamical approach to the rigidity results of R. Zimmer, whose proofs have relied on techniques derived from super-rigidity. For example, dynamical methods have been used by $\mathrm{R}$. Feres in $[12,10,11,13]$ to extend previous work of Zimmer [74] on higher rank actions. G. Stuck has mixed dynamical techniques with methods of super-rigidity for the study of codimension-one actions of semi-simple groups [63, 62, 64].

There are many other types of examples of normally hyperbolic actions. Let us give one more, which is very stupid, but still suggestive. Take any Anosov action $\varphi: \Gamma \times X \rightarrow X$, and take any compact smooth manifold $Y$ without 
boundary. Then the group $\Gamma$ also acts on the product manifold $X \times Y$, via the identity action on $Y$ !

This type of construction can be made slightly more elaborate, by considering a fibration $\pi: V \rightarrow X$ whose fibers are all diffeomorphic to $Y$. We then assume there is given an action of $\Gamma$ on the fibration $\pi: V \rightarrow X$, which is Anosov on the base space $X$. That is, $\varphi: \Gamma \times V \rightarrow V$ preserves the fibers of the map $\pi$ and induces a smooth action of the quotient $X$. This will again be normally hyperbolic.

There is another way to extend the product action, in a slightly less trivial fashion. Given an Anosov action $\varphi: \Gamma \times X \rightarrow X$, and an arbitrary smooth action of $\Gamma$ on $Y$. Suppose that these actions lift to the universal covers $\tilde{X}$ and $\tilde{Y}$ of $X$ and $Y$, respectively. (For example, the action on $X$ always lifts if $\varphi$ is a standard action.) Then there is a diagonal action of $\Gamma$ on the product of the universal coverings, $\tilde{X} \times \tilde{Y}$. Assume that there is given a group $\Lambda$ and a right action of $\Lambda$ on $\tilde{X} \times \tilde{Y}$, which commutes with the left diagonal action of $\Gamma$, so that the quotient $M=\tilde{X} \times \tilde{Y} / \Lambda$ is a compact manifold. Then there is an induced action on $M$ is normally hyperbolic. For example, the lattices $\Lambda$ investigated by Cairns and Ghys (Example 2.4, [7]) are of this type. One can modify their construction so that there is an Anosov action of a group $\Gamma$ on one of the factors, then the left and right actions will automatically commute and we obtain examples of the type under discussion.

It is expected that many of the topological rigidity results for Anosov actions carries over to normally hyperbolic actions, as this is often true for the techniques used (cf. [45, 60, 61].) For example, the following extension of Theorem 2.3 was announced in [26]:

THEOREM 6.9 Let $\pi: V \rightarrow X$ be a fibration, where $V$ and $X$ are connected manifolds without boundary. Suppose there is given a $C^{1}$-action $\varphi: \Gamma \times V \rightarrow V$ which preserves the fibers of the fibration $\pi$, and induces an Anosov $C^{1}$-action $\varphi_{0}$ on the base space $X$. Assume that the periodic points $\Lambda\left(\varphi_{0}\right)$ are dense in $X$, and $\Gamma$ satisfies the condition $S V C\left(n^{2}\right)$ where $X$ has dimension $n$. Then $\varphi$ is topologically deformation rigid.

We conclude with a short list of questions and problems:

QUESTION 6.10 1. Are there topologically trivial $C^{1}$-deformations of an Anosov action of a higher rank lattice which are not $C^{1}$-trivial?

2. Is there an affine Anosov action of a higher rank lattice without a periodic orbit?

3. Is there a classification of the affine Anosov actions of higher rank lattices? ( If one assumes there exists a periodic orbit for the action, then this amounts to calculating the torsion in the cohomology of the standard representation of a subgroup of $S L(n, \mathbf{Z})$ acting on $\mathbf{T}^{n}$.)

4. Give concrete examples of Anosov actions that arise from the restrictionof-scalars technique (Example 3). 
5. Give an example of an action of an irreducible rank one lattice in $S L(n, \mathbf{Z})$ with homological dimension greater than one, whose standard action on $\mathbf{T}^{n}$ is not deformation rigid. That is, extend the construction of Example 7 to the fundamental group $\Gamma$ of a finite-volume, negatively curved manifold of dimension greater than 2, where there is a homomorphism of $\Gamma$ into $S L(n, \mathbf{Z})$.

6. Give an example of a smooth action $\varphi$ of a higher rank lattice $\Gamma$ on a compact manifold such that there is an element $\gamma \in \Gamma$ for which $\varphi(\gamma)$ is Axiom A, but not Anosov.

\section{References}

[1] B. Anderson. Diffeomorphisms with discrete centralizer. Topology, 15:143-147, 1976.

[2] D. V. Anosov. Geodesic Flows on Closed Riemannian Manifolds with Negative Curvature, volume 90 (1967) of Proc. Steklov Inst. Math. Amer. Math. Soc., 1969.

[3] H. Bass, M. Lazard, and J.-P. Serre. Sous groupes d'indice fini dans $S L(n, \mathbf{Z})$. Bulletin Amer. Math. Soc., 70:385-392, 1964.

[4] H. Bass, J. Milnor, and J.-P. Serre. Solution of the congruence subgroup problem for $S L_{n}(n \geq 3)$ and $S p_{2 n}(n \geq 2)$. Publ. Math. Inst. Hautes Etudes Sci., 33:421499, 1967.

[5] Y. Benoist and F. Labourie. Sur les difféomorphismes d'Anosov à feuilletages stable et instable différentiables. preprint, Mars, 1992.

[6] A. Borel and Harish-Chandra. Arithmetic subgroups of algebraic groups. Annals of Math., 75:485-535, 1962.

[7] G. Cairns and E. Ghys. Totally geodesic foliations on 4-manifolds. Jour. Differential Geom., 23:241-254, 1986.

[8] E. Cawley. The Teichmüller space of the standard action of $S L(2, \mathbf{Z})$ on $\mathbf{T}^{2}$ is trivial. Duke Math. Journal, 67:135-141, 1992.

[9] S. G. Dani. Nilmanifolds with Anosov automorphism. Jour. London Math. Soc., 18:553-559, 1978.

[10] R. Feres. Affine actions of higher rank lattices. preprint, 1992.

[11] R. Feres. The center foliation of an affine diffeomorphism. Geometriae Dedicata, 1992. to appear.

[12] R. Feres. Connection-preserving actions of lattices in $S L_{n}(\mathbf{R})$. Israel Jour. Math., 1992. to appear.

[13] R. Feres. The invariant connection of a $\frac{1}{2}$-pinched Anosov diffeomorphism and rigidity. preprint, 1992.

[14] L. Flaminio and A. Katok. Rigidity of symplectic Anosov diffeomorphisms on low dimensional tori. Ergodic Theory Dynamical Systems, 11:427-440, 1990.

[15] J. Franks. Anosov diffeomorphisms on tori. Trans. Amer. Math. Soc., 145:117124,1969

[16] P. Griffiths and J. Harris. Principles of Algebraic Geometry. Wiley-Interscience, New York, 1978. 
[17] M. Gromov. Rigid transformation groups. In D. Bernard and Y. Choquet-Bruhat, editors, Géométrie et Physique, Paris, 1987. Hermann.

[18] V. Guillemin and D. Kazhdan. On the cohomology of certain dynamical systems. Topology, 19:291-299, 1980.

[19] U. Hamenstadt. Entropy-rigidity of locally symmetric spaces of negative curvature. Annals of Math., 131:35-51, 1990.

[20] B. Hasselblatt. Regularity of the Anosov splitting and of horospheric foliations. preprint, 1990.

[21] J. Heitsch. Flat bundles and residues for foliations. Invent. Math., 73:271-285, 1983.

[22] J. Heitsch. Some interesting group actions. In J. Kaminker, editor, Geometric and Topological Invariants of Elliptic Operators, pages 113-123, Providence, 1990. Amer. Math. Soc. Contemp. Math. vol. 105.

[23] S. Hurder. Global invariants for measured foliations. Trans. Amer. Math. Soc., 280:367-391, 1983

[24] S. Hurder. Problems on rigidity of group actions and cocycles. Ergodic Theory Dynamical Systems, 5:473-484, 1985.

[25] S. Hurder. Deformation rigidity for subgroups of $S L(n, \mathbf{Z})$ acting on the $n$-torus. Bulletin Amer. Math. Soc., 23:107-113, July 1990.

[26] S. Hurder. Rigidity for actions of higher rank lattices. Talk given at conference $G e-$ ometric Rigidity and Hyperbolic Dynamics. Penn. State University, March 1991.

[27] S. Hurder. Affine Anosov actions. Mich. Math. Journal, 1992. to appear.

[28] S. Hurder. Rigidity for Anosov actions of higher rank lattices. Annals of Math., 135:361-410, 1992

[29] S. Hurder. Rigidity for regular Anosov actions of higher rank lattices. preprint, 1992.

[30] S. Hurder. Topological rigidity of strong stable foliations for Cartan actions. preprint, 1992.

[31] S. Hurder and A. Katok. Differentiability, rigidity and Godbillon-Vey classes for Anosov flows. Publ. Math. Inst. Hautes Etudes Sci., 72:5-64, 1990.

[32] S. Hurder, A. Katok, J. Lewis, and R. Zimmer. Rigidity of Cartan actions of higher rank lattices. preprint, 1991.

[33] A. Katok and J. Lewis. Global rigidity results for lattice actions on tori and new examples of volume-preserving actions. preprint, 1991.

[34] A. Katok and J. Lewis. Local rigidity for certain groups of toral automorphisms. Israel Jour. Math., 75:203-241, 1991.

[35] A. Katok, J. Lewis, and R. Zimmer. Cocycle superrigidty and rigidity for lattice actions on tori. preprint, 1992.

[36] A. Katok and R. Spatzier. Differential rigidity of hyperbolic abelian actions. preprint, April, 1992.

[37] J. Lewis. Infinitesimal rigidity for the action of $S L(n, \mathbf{Z})$ on $\mathbf{T}^{n}$. Trans. Amer. Math. Soc., 234:421-445, 1991. 
[38] A. Livsic. Homology properties of U-systems. Math. Notes of U.S.S.R., 10:758$763,1971$.

[39] A. Livsic. Cohomology of dynamical systems. Math. U.S.S.R. Izvestija, 6:12781301, 1972.

[40] R. de la Llavé. Invariants for smooth conjugacy of hyperbolic dynamical systems, II. Comm. Math. Phys., 109:369-378, 1987.

[41] R. de la Llavé. Analytic regularity of solutions of Livsic's cohomology equation and some applications to analytic conjugacy of hyperbolic dynamical systems. Ergodic Theory Dynamical Systems, 1992.

[42] R. de la Llavé. Smooth conjugacy and S-R-B measures for uniformly and nonuniformly hyperbolic systems. preprint, April, 1991. IHES/M/91/30.

[43] R. de la Llavé, J. Marco, and R. Moriyon. Canonical perturbation theory of Anosov systems and regularity results for Livsic cohomology equation. Annals of Math., 123:537-612, 1986.

[44] R. de la Llavé and R. Moriyon. Invariants for smooth conjugacy of hyperbolic dynamical systems, IV. Comm. Math. Phys., 116:185-192, 1988.

[45] C. Pugh M. Hirsch and M. Shub. Invariant Manifolds, volume 583 of Lect. Notes Math. Springer-Verlag, New York and Berlin, 1977.

[46] A. Manning. There are no new Anosov diffeomorphisms on tori. American Jour. Math., 96:422-429, 1974.

[47] J. M. Marco and R. Moriyon. Invariants for smooth conjugacy of hyperbolic dynamical systems, I. Comm. Math. Phys., 109:681-689, 1987.

[48] J. M. Marco and R. Moriyon. Invariants for smooth conjugacy of hyperbolic dynamical systems, III. Comm. Math. Phys., 112:317-333, 1987.

[49] G. A. Margulis. Discrete Subgroups of Semisimple Lie Groups. Springer-Verlag, New York and Berlin, 1991.

[50] J. Mennicke. Finite factor groups of the unimodular group. Annals of Math., $81: 31-37,1965$

[51] S. Newhouse. On codimension one Anosov diffeomorphisms. American Jour. Math., 92:761-770, 1970.

[52] J. Palis and J. C. Yoccoz. Centralizers of Anosov diffeomorphisms on tori. Ann. Sci. Ecole Norm. Sup., 22:99-108, 1989.

[53] J. Palis and J. C. Yoccoz. Rigidity of centralizers of diffeomorphisms. Ann. Sci. Ecole Norm. Sup., 22:81-98, 1989.

[54] M. Pollicott. Local rigidity of actions with hyperbolic generators: The standard action of $S L(n, \mathbf{Z})$ on $\mathbf{T}^{n}$ for $n \geq 3$ (revised version). preprint, 1992.

[55] G. Prasad and M. S. Raghunathan. Cartan subgroups and lattices in semi-simple groups. Annals of Math., 96:296-317, 1972.

[56] M. S. Raghunathan. Discrete Subgroups of Lie Groups. Springer-Verlag, 1972.

[57] O. H. Rasmussen. Continuous variation of foliations in codimension two. Topology, 19:225-349, 1980.

[58] M. Shub. Global Stability of Dynamical Systems. Springer-Verlag, New York and Berlin, 1987. 
[59] S. Smale. Differentiable dynamical systems. Bulletin Amer. Math. Soc., 73:747$817,1967$.

[60] D. Stowe. The stationary set of a group action. Proc. Amer. Math. Soc., 79:139$146,1980$.

[61] D. Stowe. Stable orbits of differentiable group actions. Trans. Amer. Math. Soc., pages 665-684, 1983.

[62] G. Stuck. Locally free actions of semi-simple groups. preprint, 1991.

[63] G. Stuck. Low dimensional actions of semi-simple groups. Israel Jour. Math., 1991.

[64] G. Stuck. Growth of homogeneous spaces, density of discrete subgroups, and Kazhdan's property (T). Invent. Math., 1992. to appear.

[65] A. Weil. On discrete subgroups of Lie groups. Annals of Math., 72:369-384, 1960.

[66] A. Weil. On discrete subgroups of Lie groups, II. Annals of Math., 75:578-602, 1962.

[67] A. Weil. Remarks on the cohomology of groups. Annals of Math., 80:149-157, 1964.

[68] R. J. Zimmer. Strong rigidity for ergodic actions of semi-simple Lie groups. Annals of Math., 112:511-529, 1980.

[69] R. J. Zimmer. On the cohomology of ergodic actions of semi-simple Lie groups and discrete subgroups. American Jour. Math., 103:937-950, 1981.

[70] R. J. Zimmer. Orbit equivalence and rigidity of ergodic actions of Lie groups. Ergodic Theory Dynamical Systems, 1:237-253, 1981.

[71] R. J. Zimmer. Ergodic Theory and Semisimple Groups. Birkhäuser, Boston, Basel, Stuttgart, 1984.

[72] R. J. Zimmer. Volume preserving actions of lattices in semisimple groups on compact manifolds. Publ. Math. Inst. Hautes Etudes Sci., 59:5-33, 1984.

[73] R. J. Zimmer. Actions of semisimple groups and discrete groups. In Proc. Int. Congress Math., Berkeley, pages 1247-1258, 1986.

[74] R. J. Zimmer. On connection-preserving actions of discrete linear groups. Ergodic Theory Dynamical Systems, 6:639-644, 1986.

[75] R. J. Zimmer. Lattices in semi-simple groups and invariant geometric structures on compact manifolds. In Roger Howe, editor, Discrete Groups in Geometry and Analysis: Papers in honor of G. D. Mostow on his sixtieth birthday, Progress in Mathematics, Boston, 1987. Birkhauser.

[76] R. J. Zimmer. Infinitesimal rigidity for smooth actions of discrete subgroups of Lie groups. Jour. Differential Geom., 31:301-322, 1990.

[77] R. J. Zimmer. On the algebraic hull of an automorphism group of a principal bundle. Comment. Math. Helv., 65:375-387, 1990.

Department of Mathematics (M/C 249)

UNIVERSITY OF IllinoIs AT CHICAGO

P. O. Box 4348

CHICAGO, IL 60680

E-mail address: hurder@math.uic.edu 